\title{
Rapid spectral and timing variability of Be/X-ray binaries during type II outbursts
}

\author{
P. Reig ${ }^{1,2}$ \\ 1 IESL, Foundation for Research and Technology-Hellas, 71110, Heraklion, Greece \\ 2 Physics Department, University of Crete, 71003, Heraklion, Greece \\ e-mail: pau@physics.uoc.gr
}

Received 22 April 2008 / Accepted 27 July 2008

ABSTRACT

\begin{abstract}
X-ray colour-colour (CD) and colour-intensity (HID) diagrams are powerful tools that allow investigation of spectral variability without assuming any spectral model. These diagrams have been used extensively for low-mass X-ray binaries and black-hole candidates, but very few applications have been found for high-mass X-ray binaries. We investigated the spectral and timing variability of four accreting X-ray pulsars with Be-type companions during major X-ray outbursts. The aim is to define source states based on the properties (noise components) of the aperiodic variability in correlation with the position in the colour-colour diagram. Different spectral states were defined according to the value of the X-ray colours and flux. Transient Be/X-ray binaries exhibit two branches in their colour-colour and colour-intensity diagrams: i) the horizontal branch corresponds to a low-intensity state and shows the highest fractional rms, similar to the the island state in atolls and horizontal branch in $\mathrm{Z}$ sources; ii) the diagonal branch corresponds to a high-intensity state, in which the source stays for about $75 \%$ of the total duration of the outburst. Despite the complexity of the power spectra due to the peaks of the pulse period and its harmonics, the aperiodic variability of Be/X-ray binaries can be described with a relatively low number of Lorentzian components. Some of these components can be associated with the same type of noise as seen in low-mass X-ray binaries, although the characteristic frequencies are about one order of magnitude lower. The analysis of the CD/HID and power spectra results in two different types of Be/X. While in 4U 0115+63, KS 1947+300 and EXO 2030+375 the hard colour decreases as the count rate decreases, it increases in V0332+53. The pattern traced by V0332+53 then results in a Z-shaped track, similar to the low-mass $Z$ sources, without the flaring branch. In contrast, the horizontal branch in 4 U $0115+63$, KS $1947+300$ and EXO 2030+375 corresponds to a low/soft state, which is not seen in other types of X-ray binaries. The noise at very low frequencies follows a power law in V0332+53 (like in LMXB Z), and it is flat-topped in 4U 0115+63, KS 1947+300, and EXO $2030+375$ (like in LMXB atoll). V0332+53 shows a noise component coupled with the periodic variability that it is not seen in any of the other three sources.
\end{abstract}

Key words. stars: emission-line, $\mathrm{Be}$ - stars: neutron - X-rays: binaries - stars: pulsars: general

\section{Introduction}

The colour-colour (CD) and hardness-intensity (HID) diagrams are very useful tools to investigate the spectral variations of an $\mathrm{X}$-ray source. They are model- and instrument-independent and reflect the intrinsic properties of the system. The first application of this type of plots was the separation of different types of galactic X-ray sources and the classification of X-ray populations. The spectral hardness was defined by the ratio of counting rates in each of the proportional counters of the MIT OSO-7 experiment (Markert et al. 1977; Rappaport et al. 1977). White \& Marshall (1984) made use of the X-ray colour-colour diagrams, obtained from HEAO 1 observations, to study the spectral states among the different types of X-ray binaries, including black-hole candidates.

Colour-colour diagrams became widely used during the EXOSAT era, which also marks the introduction of HIDs (Priedhorsky et al. 1986; van der Klis et al. 1987a; Hasinger 1987), where "hardness" refers to the ratio of the count rate in two energy bands. Before EXOSAT, CDs contained sources of different origin. The novelty of the EXOSAT CDs and HIDs was that they were applied to individual sources. CD/HIDs became a useful way to investigate the rapid aperiodic variability in correlation with spectral states, as defined by the spectral hardness, of individual low-mass X-ray binaries and black-hole candidates (Schulz et al. 1989; Hasinger \& van der Klis 1989).

With the advent of RXTE the use of CDs and HIDs have become part of the standard data analysis. RXTE has provided an unprecedentedly large database of X-ray observations with exceptionally good timing and moderate spectral resolution. Together with the functional form of the variability components ("noise"), CD and HID have become an essential tool in developing a whole new phenomenology on the spectral and timing properties of X-ray binaries by introducing the notion of source states. A state is defined by the appearance of a spectral (i.e. power-law, blackbody) or variability component (i.e. Lorentzian) associated with a particular and well-defined position of the source in the CD/HID. A transition between states is assumed when the relative strength of the spectral or variability components varies and the source motion in the CD/HID changes direction (van der Klis 2006).

While there are numerous references in the literature on the application of CD/HID analysis on low-mass X-ray binaries (van der Klis 2006, and references therein) and black-hole systems (see e.g. Belloni et al. 2005), very little work of this type has been done on high-mass X-ray binaries. Belloni \& Hasinger (1990) performed an aperiodic variability study of 12 high-mass $\mathrm{X}$-ray binaries from EXOSAT archive data. The sample included 
EXO 2030+375 and V0332+53. However, the few observations analysed of $\mathrm{V} 0332+53$ did not correspond to a major X-ray outburst, while the data of EXO 2030+375 covered the decay of the 1985 outburst only partially. The other 10 sources were supergiant X-ray binaries in bright states. The vast majority of the rapid aperiodic variability studies in accreting X-ray pulsars have concentrated on the detection of QPOs (Angelini et al. 1989; Jernigan et al. 2000; Qu et al. 2005); see also Shirakawa \& Lai (2002, and references therein)

Massive X-ray binaries are classified according to the luminosity class of the optical component into $\mathrm{Be} / \mathrm{X}$-ray binaries (dwarf or subgiant) and supergiant (luminosity class I-II) X-ray binaries. The former tend to be transient systems while the latter are persistent sources. Be stars are non-supergiant fast-rotating B-type and luminosity class III-V stars which at some point of their lives have shown spectral lines in emission. In the infrared they are brighter than their non-emitting counterparts of the same spectral type. The line emission and infrared excess originate in extended circumstellar envelopes of ionized gas surrounding the equator of the B star. When the Be star takes part on a binary system where the companion is a neutron star, then the system is referred to as a Be/X-ray binary.

$\mathrm{Be} / \mathrm{X}$-ray binaries are variable on time scales from seconds to years. The fastest variability is found in the X-ray band. Virtually all the Be/X-ray binaries with identified optical counterparts are $\mathrm{X}$-ray pulsars. Pulse periods cover the range $1-10^{3} \mathrm{~s}$. On longer time scales (months to years) the variability is also apparent in the optical and infrared bands and it is attributed to structural changes of the circumstellar disc. The long-term X-ray variability of the transient $\mathrm{Be} / \mathrm{X}$-ray binaries is characterised by two type of outbursting activity:

- type I outbursts. These are regular and (quasi)periodic outbursts, normally peaking at or close to periastron passage of the neutron star. They are short-lived, i.e., tend to cover a relatively small fraction of the orbital period (typically $0.2-0.3 P_{\text {orb }}$ ). The X-ray flux increases by about one or two order of magnitude with respect to the pre-outburst state, reaching peak luminosities $L_{\mathrm{x}} \leq 10^{37} \mathrm{erg} \mathrm{s}^{-1}$;

- type II outbursts represent major increases of the X-ray flux, $10^{3}-10^{4}$ times that at quiescence. They reach the Eddington luminosity for a neutron star $\left(\sim 10^{38} \mathrm{erg} \mathrm{s}^{-1}\right)$ and become the brightest objects of the X-ray sky. They do not show any preferred orbital phase and last for a large fraction of an orbital period or even for several orbital periods. The formation of an accretion disc during type II outbursts (Kriss et al. 1983; Motch etal 1991; Hayasaki \& Ozakaki 2004; Wilson et al. 2008) may occur. The discovery of quasi-periodic oscillations in some systems (Angelini et al. 1989; Takeshima et al. 1994; Finger et al. 1996) would support this scenario. The presence of an accretion disc also helps explain the high and steady spin-up rates seen during the giant outbursts, which are difficult to account for by means of direct accretion.

In this work we present a systematic study of the aperiodic variability of four $\mathrm{Be} / \mathrm{X}$-ray binaries during type II outbursts. First, we give a summary of the relevant information of the sources. Section 3 summarises the observations and the characteristics of the instruments used in the analysis. Section 4 refers to the reduction of the data and describes how the CD/HID and power spectra were obtained. A detailed description of the results is given in Sect. 5. This section is divided into three subsections describing the outburst profiles, the colour analysis and the aperiodic variability. Our interpretation of the results and the implications of this work are discussed in Sect. 6. Final conclusions are drawn in Sect. 7.

\section{The sources}

\section{1. $4 \cup 0115+63$}

$4 \mathrm{U} 0115+63$ is one of the most active and best studied Be/X-ray transients as it was one of the first $\mathrm{Be} / \mathrm{X}$-ray binaries to be discovered. The oldest available X-ray observations date back to August 1969 when the Vela 5B satellite detected the source as three small outbursts separated by 180 days (Whitlock 1989). Since then about 15 outbursts have been reported (Reig et al. 2007). During the RXTE's life time 3 major outbursts have been detected: in 1999, 2000 and 2004. Here we analyse observations from the 2004 event. The neutron star orbits a B0.2Ve in a moderately eccentric orbit with $P_{\text {orb }}=24.3 \mathrm{~d}, e=0.34$ and $a_{\mathrm{x}} \sin i=140.1$ lt-s (Rappaport et al. 1978) and rotates with $P_{\text {spin }}=3.6 \mathrm{~s}$, as inferred from the pulsed X-ray emission. The distance to the source is estimated to be $\sim 8 \mathrm{kpc}$ (Reig et al. 2007).

The X-ray spectrum shows up to five cyclotron resonance scattering features (fundamental and four harmonics) and constitutes the accreting neutron star with the highest number of cyclotron lines (Heindl et al. 2004). The optical and infrared emission is characterised by cyclic changes with a period of $\sim 5$ years. Another peculiarity is that X-ray outbursts in 4U 0115+63 appear to come in pairs, i.e., two in every cycle. However, sometimes the second outburst is missing (Reig et al. 2007). mHZ QPOs were detected during the 1999 and 2004 outbursts (Heindl et al. 1999; Coburn et al. 2004).

\subsection{KS $1947+300$}

The Be/X-ray binary KS $1947+300$ was first detected in the X-ray band on June 8, 1989 by the TTM coded mask X-ray spectrograph on the Kvant module of the Mir space station (Borozdin et al. 1990) with a peak flux of 70 mCrab. About 35 days later the flux was at the limit of sensitivity of the instrument with a $3 \sigma$ upper limit of $10 \mathrm{mCrab}$. X-ray pulsations with a pulse period of $18.7 \mathrm{~s}$ were found in the X-ray flux of GRO J1948+32 in April 1994 by the all-sky monitor BATSE aboard CGRO (Chakrabarty et al. 1995). The source reached a peak pulse flux of $50 \mathrm{mCrab}$ in the energy range $20-75 \mathrm{keV}$. About 25 days later the flux decayed bellow the detection threshold of BATSE. Given the very large error box of GRO J1948+32 no connection with this source and KS 1947+300 was realised. The source remained in quiescence until the beginning of 2001 (Galloway et al. 2004). In this occasion the source reached a maximum flux of $120 \mathrm{mCrab}$, the highest in its history. This outburst is the object of the present work. Prior to this major outburst, weak emission ( $\sim 20 \mathrm{mCrab})$ and X-ray pulsations $(18.7579 \pm 0.0005 \mathrm{~s})$ from KS $1947+300$ had been detected in 2000 November (Levine \& Corbet 2000; Swank \& Morgan 2000). The coincidence in the value of the pulse period and the location of KS 1947+300 inside the GRO J1948+32 error circle suggest that the two sources are in fact the same object. Pulse timing analysis allowed to solve for the orbital parameters resulting in orbital period $P_{\text {orb }}=40.415 \pm 0.010 \mathrm{~d}$, eccentricity $e=0.033 \pm 0.013$ and projected radius $a_{\mathrm{x}} \sin i=137 \pm 3$ 1t-s (Galloway et al. 2004). A broad-band spectral study of KS 1947+300 using BeppoSAX observations (Naik et al. 2006) showed that the energy spectrum in the $0.1-100 \mathrm{keV}$ energy band has three components: a Comptonized component, a $\sim 0.6 \mathrm{keV}$ 
blackbody component, and a narrow, weak iron emission line at $6.7 \mathrm{keV}$ with a low column density of material in the line of sight. Although the optical counterpart to KS 1947+300 was correctly identified soon after the Kvant detection (Goranskii et al. 1991; Grankin et al. 1991) its identification as a Be/X-ray binary was suggested by Negueruela et al. (2003). KS 1947+300 is associated with a moderately reddened $V=14.2$ B0Ve star located at $\sim 10 \mathrm{kpc}$.

\subsection{EXO $2030+375$}

EXO 2030+375 was discovered by EXOSAT in 1985 (Parmar et al. 1989). Since then it has been extensively observed in the $\mathrm{X} / \gamma$-ray band by various missions: EXOSAT (Reynolds et al. 1993), ROSAT (Mavromatakis 1994), RXTE (Reig \& Coe 1998, 1999), CGRO (Stollber et al. 1999), INTEGRAL (Camero-Arranz et al. 2005) and SWIFT (Klochkov et al. 2007). EXO $2030+375$ is the prototype of X-ray variability in Be/X-ray binaries. It shows a regular pattern of type I outbursts $\left(L_{\mathrm{X}} \approx\right.$ $10^{36} \mathrm{erg} \mathrm{s}^{-1}$ ) during each periastron passage (Wilson et al. 2002). In addition, EXO $2030+375$ has shown two major outbursts $\left(L_{\mathrm{x}} \gtrsim 10^{38} \mathrm{erg} \mathrm{s}^{-1}\right)$ : the first one in 1985 that led to its discovery as an X-ray transient and the second one in 2006. In this work we analyse RXTE observations of the latest event.

EXO 2030+375 contains a rotating neutron star $\left(P_{\text {spin }}=\right.$ 41.7 s) orbiting a Be primary (Motch \& Janot-Pacheco 1987; Coe et al. 1988; Reig et al. 1998) in a moderately eccentric $(e=0.4)$ wide $\left(P_{\text {orb }}=46 \mathrm{~d}\right)$ orbit and is located at about $7.1 \mathrm{kpc}$ (Wilson et al. 2002). Quasi-periodic oscillations with a frequency of $0.2 \mathrm{~Hz}$ were detected from this source when the luminosity was close to its maximum during the 1985 outburst (Angelini et al. 1989). In common with other high-mass X-ray binaries, the continuum spectral shape in the range $1-30 \mathrm{keV}$ can be represented by a power law with an exponential cutoff (Reynolds et al. 1993). A blackbody component has been reported to give good fits at very high $\left(L_{\mathrm{x}} \sim 10^{38} \mathrm{erg} \mathrm{s}^{-1}\right)$ luminosity (Sun et al. 1994) and relatively low $\left(L_{\mathrm{x}} \approx 10^{36} \mathrm{erg} \mathrm{s}^{-1}\right)$ luminosity (Reig \& Coe 1999).

\section{4. $V 0332+53$}

A similar analysis to the one presented here has already been published in Reig et al. (2006). For the sake of clarity and in order to apply the same reduction procedure to all sources, we have re-analysed the observations in Reig et al. (2006). Note also that the present work includes data during the rise of the outburst that were not previously analysed and that the frequency range of the power spectra is two orders of magnitude larger than in Reig et al. (2006).

$\mathrm{V} 0332+53$ is a hard X-ray transient that spends most of its life in a quiescent $X$-ray state. After more than 15 years of quiescence the $\mathrm{Be} / \mathrm{X}$-ray binary $\mathrm{V} 0332+53$ underwent a giant outburst in December 2004. The outburst was predicted about 9 months before its occurrence from the brightening of the optical companion (Goranskij \& Barsukova 2004). Prior to the event reported here, V0332+53 had went into outburst is three other occasions. The 1973 outburst led to its discovery as a bright X-ray source by the Vela $5 B$ satellite (Terrell \& Priedhorsky 1984). In 1983 it reappeared in the form of three small outbursts (Tanaka 1983). EXOSAT observations of this activity period resulted in the discovery of X-ray pulsations with $P_{\text {spin }}=4.4 \mathrm{~s}$ and the determination of the orbital parameters, $P_{\text {orb }}=34.25 \mathrm{~d}$ and $e=0.31$ (Stella et al. 1985). In 1989 Ginga detected V0332+53 again and allowed the discovery of a cyclotron resonant scattering feature at $28.5 \mathrm{keV}$ (Makishima et al. 1990) and QPOs at $0.051 \mathrm{~Hz}$ (Takeshima et al. 1994). The optical counterpart to $\mathrm{V} 0332+53$ is an O8-9Ve star at a distance of $\sim 7 \mathrm{kpc}$, showing $\mathrm{H} \alpha$ in emission and strong and variable infrared emission (Bernacca et al. 1984; Corbet et al. 1986; Coe et al. 1987; Negueruela et al. 1999).

\section{Observations}

We analysed data obtained by the Proportional Counter Array (PCA) and the All Sky Monitor (ASM) onboard the Rossi X-ray Timing Explorer (RXTE). The ASM data consist of daily flux averages in the energy range $1.3-12.1 \mathrm{keV}$. The PCA covers the lower part of the energy range $2-60 \mathrm{keV}$, and consists of five identical coaligned gas-filled proportional units. Due to RXTE's low-Earth orbit, the data consist of a number of contiguous data intervals (typically $1 \mathrm{~h}$ long) interspersed with observational gaps produced by Earth occultations of the source and passages of the satellite through the South Atlantic Anomaly. We shall refer to each of these data intervals as "pointings". Data taken during satellite slews, passage through the South Atlantic Anomaly and Earth occultation were removed.

During the RXTE life time the response of the detectors varied due to ageing. Also, gain changes are applied occasionally, making the channel boundaries for a given energy range change with time, and also slightly affecting the effective areas of the detectors. Each gain change is the start of a new "gain epoch". To minimize this effect, data from the same "epoch" was used. All the data analysed in this work correspond to "epoch" 5. In addition, the source X-ray colours were normalised to those of the $\mathrm{Crab}$ that are closest in time. Table 2 gives relevant information about the observations.

\section{Data reduction and analysis}

In this section we describe the different tools (CD/HID and power spectra) used in the data analysis. All Sky Monior (ASM) light curves were obtained to study the outburst profiles. Data from the PCA can be collected and telemetered to the ground in many different ways depending on the intensity of the source and the spectral and timing resolution desired. In this work we used Standard2 to obtain colour-colour and hardness-intensity diagrams and time-average energy spectra. In addition, various high-time resolution modes (good_xenon,

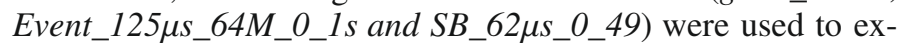
tract the light curves with which the power spectra were produced.

\subsection{Outburst profiles}

The outburst profiles were obtained from the 1-day binned ASM light curves and are shown in Fig. 1. Table 1 gives the duration and peak intensity of each individual outburst together with some relevant information about the systems. The time of the PCA observations are marked in Fig. 1 with different colours indicating different spectral regions (see the electronic edition of the Journal for a colour version of this figure). Each vertical mark corresponds to a pointing. In the energy range of the ASM, 1 Crab corresponds to $\sim 75 \mathrm{c} \mathrm{s}^{-1}$. In general, the PCA sampling of the outbursts is excellent. The only part of the outburst not covered by the PCA data is the 7-10 first days, which can be attributed to the transient nature of the sources (it takes some time 

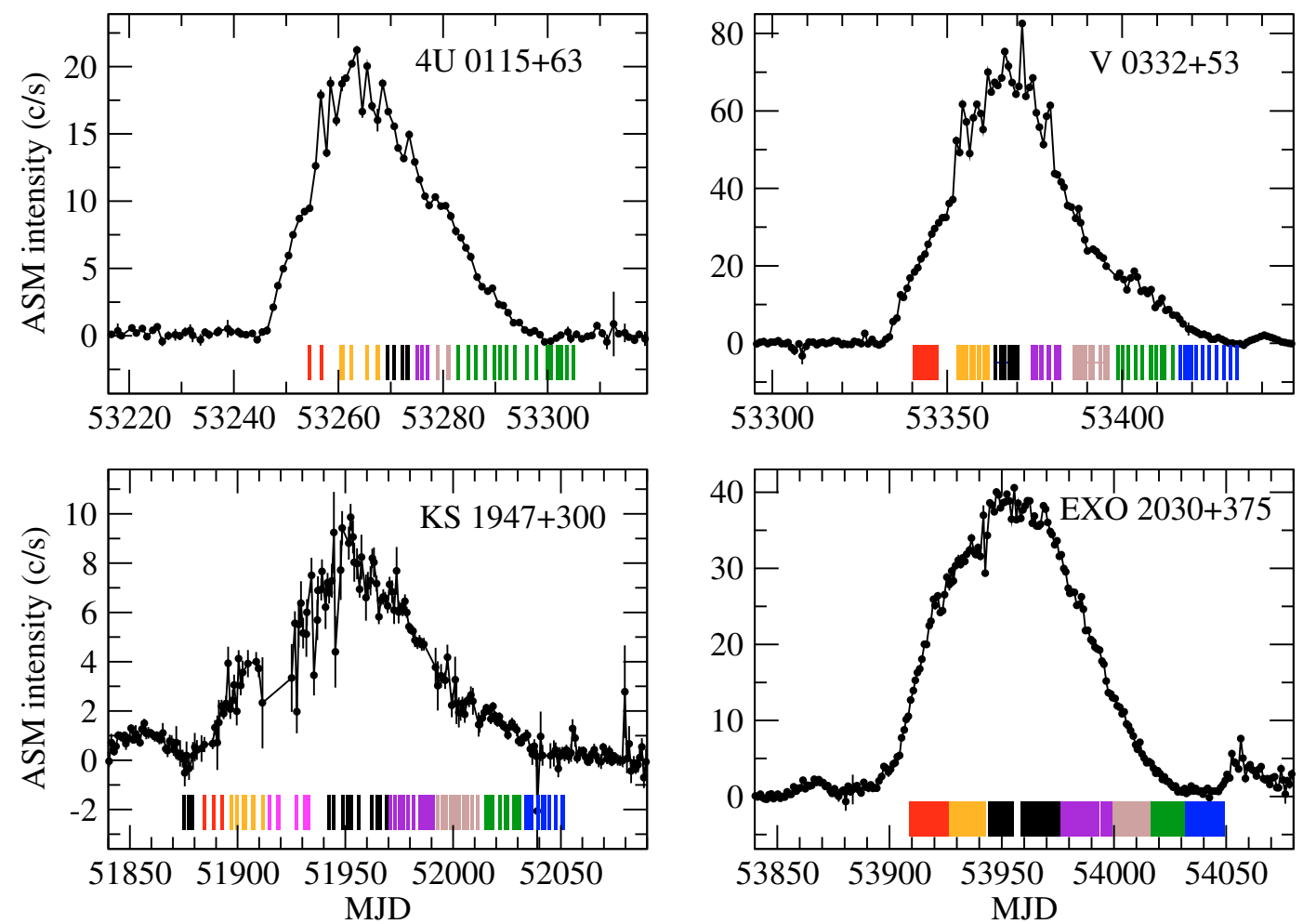

Fig. 1. The outburst profiles as seen by ASM RXTE. Vertical lines mark the time of the RXTE PCA observations. Different colours distinguish the spectral regions. Bin size is 1 day. 1 Crab corresponds to $\sim 75 \mathrm{c} / \mathrm{s}$. See the electronic edition of the Journal for a colour version of this figure.

Table 1. Optical and X-ray information of the systems and the outbursts analysed in this work.

\begin{tabular}{lcccccccc}
\hline \hline $\begin{array}{l}\text { Source } \\
\text { name }\end{array}$ & $\begin{array}{c}\text { Spectral } \\
\text { type }\end{array}$ & $\begin{array}{c}P_{\text {spin }} \\
(\mathrm{s})\end{array}$ & $\begin{array}{c}P_{\text {orb }} \\
(\mathrm{d})\end{array}$ & $e$ & $\begin{array}{c}\text { Distance } \\
(\mathrm{kpc})\end{array}$ & $\begin{array}{c}\text { Outburst } \\
\text { duration }(\mathrm{d})\end{array}$ & $\begin{array}{c}L_{\mathrm{X}, \text { peak }} \\
\text { 3-30 keV }\left(\mathrm{erg} \mathrm{s}^{-1}\right)\end{array}$ & $\begin{array}{c}\text { On-source } \\
\text { time }(\mathrm{ks})\end{array}$ \\
\hline 4U 0115+63 & B0.2V & 3.6 & 24.3 & 0.34 & 8.1 & 55 & $1.2 \times 10^{38}$ & 91 \\
KS 1947+300 & B0V & 18.7 & 40.4 & 0.03 & 10 & 165 & $6.2 \times 10^{37}$ & 132 \\
EXO 2030+375 & B0.5III-V & 41.8 & 46.0 & 0.41 & 7.1 & 155 & $1.4 \times 10^{38}$ & 166 \\
V 0332+53 & O8-9V & 4.4 & 34.2 & 0.30 & 7 & 105 & $3.2 \times 10^{38}$ & 198 \\
\hline
\end{tabular}

to issue an alert warning and reschedule the observations). The only source with available data covering the entire outburst is KS $1947+300$.

\subsection{Colour-colour and hardness-intensity diagrams}

Colour-colour (CD) and hardness-intensity (HID) diagrams are powerful tools that allow the investigation of spectral variability without the assumption of any spectral model. In order to study the evolution of the spectral shape as the outburst evolves, the RXTE light curve was divided into different parts depending on count rate and whether the outburst was on the rise, peak or decay. Given the good sampling of the PCA observations, these three phases were further subdivided into shorter intervals. Thus each interval defines a spectral region characterised by count rate, soft and hard colours. Table 2 gives relevant information about each region and source.

The CD and HID were obtained from the backgroundsubtracted Standard 2 PCA light curves in the energy ranges $c_{1}=4-7 \mathrm{keV}, c_{2}=7-10 \mathrm{keV}, c_{3}=10-15 \mathrm{keV}$ and $c_{4}=15-$ $30 \mathrm{keV}$. The soft colour (SC) was defined as the ratio $c_{2} / c_{1}$ and the hard colour (HC) as the ratio $c_{4} / c_{3}$. These ratios are expected to be insensitive to interstellar absorption effects. The hydrogen column densities to the systems, obtained from model fits to the
$\mathrm{X}$-ray spectra, are in the range $0.6-3 \times 10^{22} \mathrm{~cm}^{-2}$. The evolution with time of the PCU2 count rate and the soft and hard colours is shown in Fig. 2.

The CD was constructed by plotting the hard colour as function of the soft colour (Fig. 3) and the HID by plotting the count rate in the 4-30 keV band as a function of one of the colours (Fig. 4). To recover the true values of the colours of the sources, the soft colour should be multiplied by 0.34 and the hard color by 0.47 (quoted values are averages of the Crab colours during the observations). The variation of the Crab colours computed as the root-mean-square, i.e. the standard deviation over the mean colour, was, on average, $2.1 \%$ and $1.6 \%$ for the soft and hard colours, respectively.

To reduce the uncertainty in the individual 16-s points (default resolution of the Standard 2 mode) the light curves were rebinned to 512-s bins. Also, data for which the resulting relative errors were larger than $5 \%$ were excluded. When the count rate per PCU was lower than $10 \mathrm{c} \mathrm{s}^{-1}$, relative errors up to $10 \%$ were allowed.

\subsection{Power spectral analysis}

Power spectra were created by performing a fast Fourier transform on the light curves according to the following 
Table 2. Observations and definition of source states.

\begin{tabular}{|c|c|c|c|c|c|c|c|c|}
\hline $\begin{array}{l}\text { Source } \\
\text { state }\end{array}$ & Branch $^{a}$ & $\begin{array}{c}\text { Time range } \\
\text { MJD }\end{array}$ & $\begin{array}{l}\text { Proposal } \\
\text { ID }\end{array}$ & $\begin{array}{c}\text { On-source } \\
\text { time }(\mathrm{s})\end{array}$ & $\langle S C\rangle^{b}$ & $\langle H C\rangle^{b}$ & Flux $^{c}$ & Luminosity $^{d}$ \\
\hline \multicolumn{9}{|c|}{$4 \mathrm{U} 0115+63$} \\
\hline Rise1 & DB & $53254.0676-53256.4660$ & 90089 & 3872 & $2.08 \pm 0.05$ & $0.71 \pm 0.03$ & 0.95 & 7.5 \\
\hline Rise2 & DB & $53260.1495-53265.0921$ & 90089 & 3536 & $1.91 \pm 0.05$ & $0.81 \pm 0.04$ & 1.5 & 11.8 \\
\hline Peak & DB & $53267.1686-53272.9721$ & 90089 & 29744 & $1.87 \pm 0.01$ & $0.89 \pm 0.01$ & 1.4 & 11.0 \\
\hline Decay1 & DB & $53274.8282-53280.7687$ & 90089 & 9712 & $1.95 \pm 0.02$ & $0.85 \pm 0.02$ & 1.0 & 7.9 \\
\hline Decay2 & DB & $53278.6230-53280.7687$ & 90089 & 6128 & $2.03 \pm 0.04$ & $0.70 \pm 0.06$ & 0.8 & 6.0 \\
\hline Decay3 & DB & $53282.5715-53282.6673$ & 90014 & 5536 & $2.09 \pm 0.01$ & $0.62 \pm 0.01$ & 0.6 & 5.0 \\
\hline Decay4 & $\mathrm{HB}$ & $53284.6063-53304.5730$ & 90014 & 32500 & $1.99 \pm 0.04$ & $0.57 \pm 0.03$ & 0.11 & 0.9 \\
\hline \multicolumn{9}{|c|}{ KS $1947+300$} \\
\hline Rise0 & $\mathrm{HB}$ & $51874.1502-51878.3595$ & 50425 & 16720 & $1.57 \pm 0.07$ & $1.24 \pm 0.02$ & 0.01 & 0.12 \\
\hline Rise1 & DB & $51883.8286-51892.2963$ & 50425 & 14060 & $1.68 \pm 0.04$ & $1.13 \pm 0.05$ & 0.09 & 1.0 \\
\hline Rise2 & DB & $51896.1987-51910.9382$ & 50425 & 17744 & $1.55 \pm 0.03$ & $1.14 \pm 0.02$ & 0.25 & 2.3 \\
\hline Rise3 & DB & $51914.1750-51932.3484$ & 50425 & 10080 & $1.49 \pm 0.03$ & $1.16 \pm 0.03$ & 0.36 & 4.3 \\
\hline Peak & DB & $51941.6795-51968.5845$ & 50425 & 23344 & $1.40 \pm 0.02$ & $1.20 \pm 0.02$ & 0.53 & 6.3 \\
\hline Decay1 & DB & $51970.2073-51990.1352$ & 60402 & 15536 & $1.51 \pm 0.03$ & $1.19 \pm 0.02$ & 0.39 & 4.7 \\
\hline Decay2 & DB & $51992.1719-52010.8263$ & 60402 & 9888 & $1.59 \pm 0.02$ & $1.16 \pm 0.02$ & 0.22 & 2.6 \\
\hline Decay3 & DB & $52014.3856-52050.1873$ & 60402 & 9984 & $1.65 \pm 0.04$ & $1.05 \pm 0.07$ & 0.11 & 1.3 \\
\hline Decay4 & $\mathrm{HB}$ & $52052.9510-52078.1324$ & 60402 & 14720 & $1.32 \pm 0.08$ & $1.11 \pm 0.04$ & 0.008 & 0.1 \\
\hline \multicolumn{9}{|c|}{ EXO 2030+375 } \\
\hline Rise1 & DB & $53908.6156-53924.7247$ & $91089 \& 92067$ & 43200 & $1.39 \pm 0.07$ & $1.09 \pm 0.02$ & 1.4 & 8.4 \\
\hline Rise2 & DB & $53926.6282-53943.2576$ & 91089 & 26512 & $1.26 \pm 0.02$ & $1.12 \pm 0.01$ & 2.1 & 12.7 \\
\hline Peak & DB & $53944.0532-53975.4312$ & 91089 & 35536 & $1.23 \pm 0.02$ & $1.13 \pm 0.02$ & 2.4 & 14.5 \\
\hline Decay1 & DB & $53976.1186-53998.6782$ & 91089 & 25168 & $1.32 \pm 0.05$ & $1.09 \pm 0.03$ & 1.6 & 9.7 \\
\hline Decay2 & DB & $53999.5902-54015.4534$ & 92067 & 8656 & $1.47 \pm 0.05$ & $0.99 \pm 0.02$ & 0.57 & 3.4 \\
\hline Decay3 & DB & $54016.5928-54030.9436$ & 92067 & 10912 & $1.43 \pm 0.03$ & $1.02 \pm 0.04$ & 0.11 & 0.69 \\
\hline Decay4 & $\mathrm{HB}$ & $54031.7791-54047.9337$ & 92067 & 16144 & $1.41 \pm 0.03$ & $0.96 \pm 0.03$ & 0.06 & 0.37 \\
\hline \multicolumn{9}{|c|}{ V0332+53 } \\
\hline Rise1 & DB & 53 40.2869-53 346.7436 & 90089 & 48752 & $2.18 \pm 0.02$ & $0.89 \pm 0.03$ & 2.3 & 13.5 \\
\hline Rise2 & DB & $53352.7706-53361.1469$ & 90089 & 32720 & $2.06 \pm 0.03$ & $0.73 \pm 0.02$ & 5.0 & 29.3 \\
\hline Peak & DB & $53364.9113-53369.5965$ & $90089 \& 90427$ & 13040 & $1.95 \pm 0.02$ & $0.66 \pm 0.01$ & 5.5 & 32.2 \\
\hline Decay1 & DB & $53374.0267-53381.6610$ & $90427 \& 90014$ & 23504 & $2.04 \pm 0.02$ & $0.74 \pm 0.01$ & 4.2 & 24.6 \\
\hline Decay2 & DB & $53385.8724-53395.4413$ & 90014 & 38672 & $2.11 \pm 0.03$ & $0.83 \pm 0.03$ & 2.6 & 15.2 \\
\hline Decay3 & DB & $53398.4560-53414.0726$ & 90014 & 21296 & $2.20 \pm 0.04$ & $0.94 \pm 0.03$ & 1.5 & 8.8 \\
\hline Decay4 & $\mathrm{HB}$ & $53416.0726-53424.4139$ & 90014 & 20160 & $2.05 \pm 0.15$ & $1.13 \pm 0.05$ & 0.29 & 1.7 \\
\hline
\end{tabular}

${ }^{a}$ : HB: Low-intensity (Horizontal) Branch; DB: high-intensity (Diagonal) branch.

${ }^{b}$ : Normalised to the Crab. Average Crab values are $\langle S C-\rangle_{\text {Crab }}=0.34$ and $\langle H C\rangle_{\text {Crab }}=0.47$.

c: $\times 10^{-8} \mathrm{erg} \mathrm{cm}^{-2} \mathrm{~s}^{-1}$ in the $3-30 \mathrm{keV}$ band.

${ }^{d}: \times 10^{37} \mathrm{erg} \mathrm{s}^{-1}$ in the $3-30 \mathrm{keV}$ band.

procedure: one light curve in the energy range 2-20 keV (channels 0-49) was extracted for each spectral region with a time resolution of $2^{-8} \mathrm{~s}$. Each light curve was divided into 512-s segments and an FFT was calculated for each segment. The final power spectra resulted after averaging all the individual power spectra and rebinning logarithmically in frequency. All power spectra are presented in the rms normalisation, where the power integrated over a certain frequency interval equals the squared rms of the source (Belloni \& Hasinger 1990; Miyamoto et al. 1991).

The plots of the power spectra are shown in the $v \times P_{v}$ representation, where each power is multiplied by the corresponding frequency. This representation helps visualize at which frequency the contribution to the total rms variability is maximum (Belloni et al. 2002). Note that the frequency at which maximum power is attained in the $v \times P_{v}$ representation does not equal the centroid frequency of the Lorentzian, $v_{0}$, but $v_{\max }=$ $\left(v_{0}^{2}+(F W H M / 2)^{2}\right)^{1 / 2}$, where $F W H M$ is the Lorentzian fullwidth at half maximum.

The statistical Poisson noise $\left(P_{\mathrm{N}}=2 / R_{\mathrm{T}}\right.$, where $R_{\mathrm{T}}$ is the source count rate) was modified by dead time effects. Deadtime effects are expected to affect the power spectra at high frequencies, especially during the high-flux states, where the count rate is over $\sim 1000 \mathrm{c} \mathrm{s}^{-1}$. One way of checking whether the dead-time effects are important is by measuring the power at high frequencies in a Leahy normalised power spectrum. The expected power density should be at a level of 2 (Leahy et al. 1983; van der Klis 1989; Jernigan et al. 2000). As an example, the power density of EXO $2030+375$ during the peak of the outburst ("peak"), calculated for $v>100 \mathrm{~Hz}$, is 1.935 , while that obtained during the end of the outburst ("decay3") is 1.995. The correction of the Poisson noise $P_{\mathrm{N}}(v)$ for dead-time effects was calculated as described in Nowak et al. (1999).

The study of the rapid aperiodic variability in X-ray pulsars is hampered by the peaks due to the periodic pulsations. The spin period and its harmonics show up as narrow peaks in the power spectrum that distort the continuum. The width of these peaks depends on the frequency resolution, which in turn, depends on the length of the segments $(\Delta v=1 / T$, where $T$ is the total duration of the segment). On one hand, a high frequency resolution is required in order to have well defined peaks. On the other hand, higher frequency resolution implies less number of power spectra to average and worse signal-to-noise, especially at higher frequencies. As mentioned above we found a good compromise with $T=512 \mathrm{~s}$. 

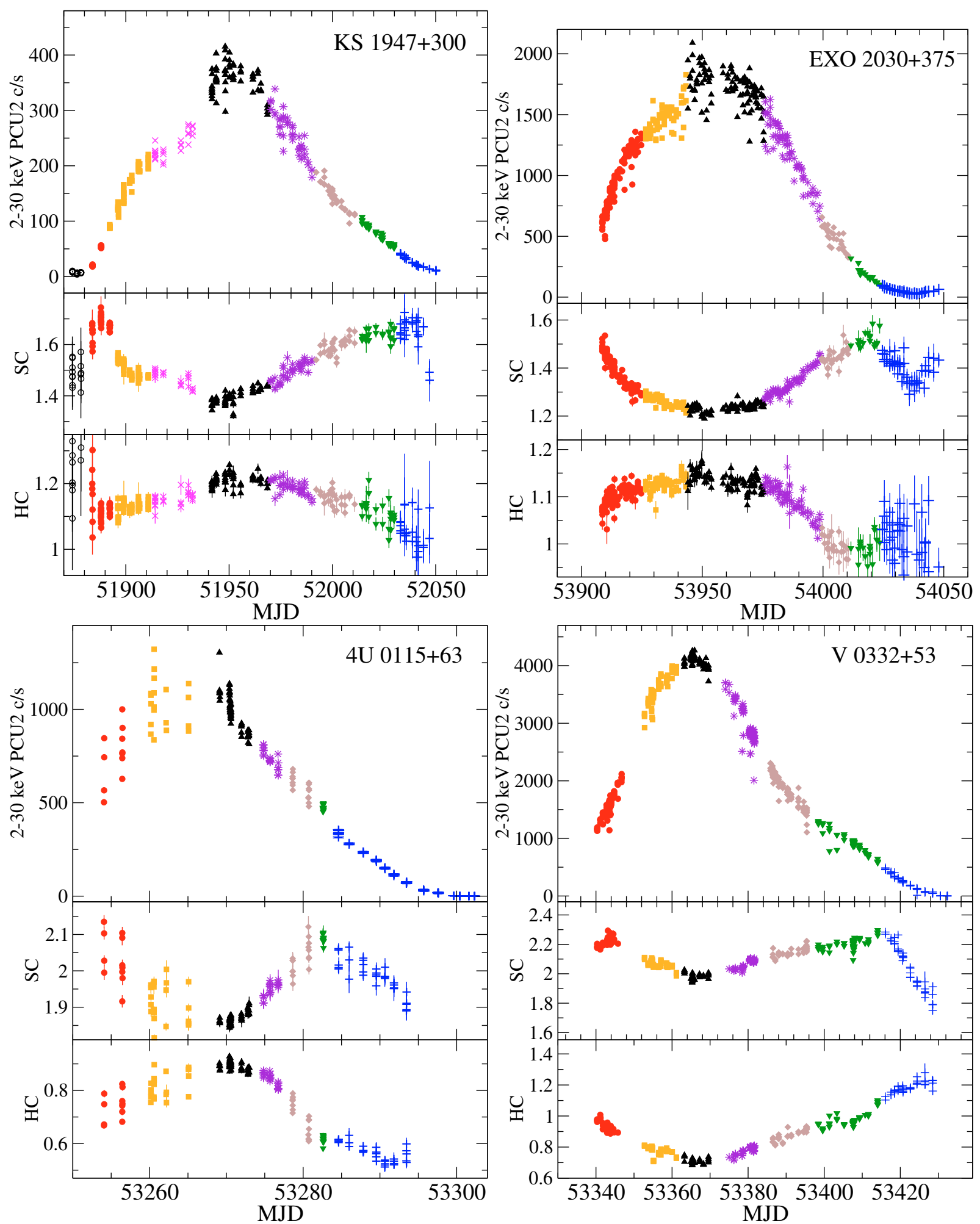

Fig. 2. PCA light curves and hardness ratio evolution. Each point represents a 512-s integration. Symbols are as follow: pre-outburst (open black circles, only in KS 1947+300), rise1 (red circles), rise2 (orange squares), rise3 (pink crosses, only in KS 1947+300), peak (black triangle-up), decay1 (violet stars), decay2 (brown diamonds), decay3 (green triangle-down) and decay4 (blue pluses). See the electronic edition of the Journal for a colour version of this figure.

Multiple Lorentzian profiles provide a good description of the power spectra in low-mass accreting neutron star binaries (see e.g. van Straaten et al. 2002, 2003; Reig et al. 2004) and black-hole systems (see e.g. Nowak 2000; Belloni et al. 2002; Pottschmidt et al. 2003). To facilitate the comparison between the noise components and timing rapid variability of all types of X-ray binaries we have followed this approach here too. The peaks from the pulsations were fitted to Lorentzian functions with fixed frequency (at the expected value according to the spin period and its harmonics) and width 

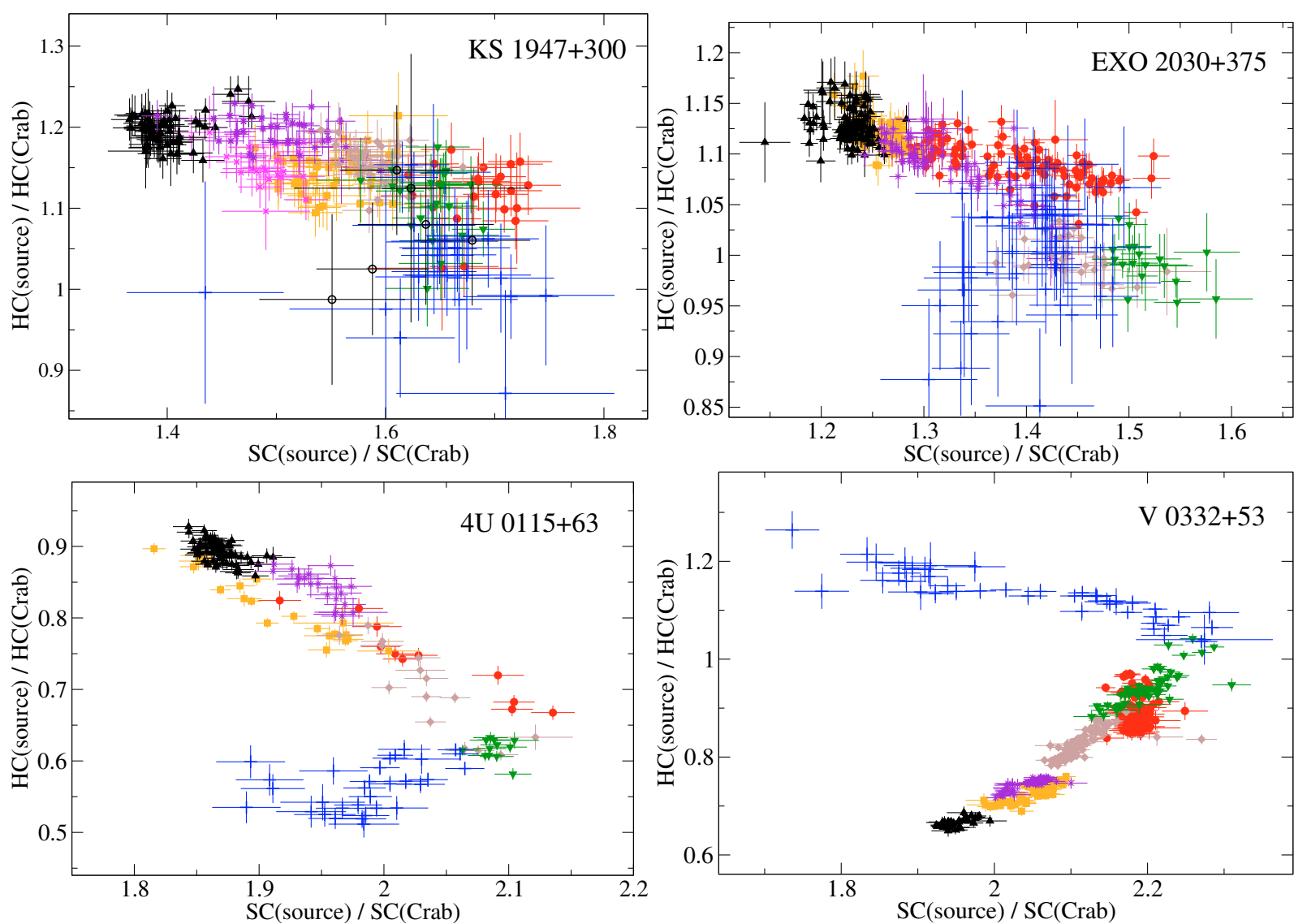

Fig. 3. Colour-colour diagrams of the four sources investigated in this work. Each point represents a 512-s integration. $S C=7-10 \mathrm{keV} / 4-7 \mathrm{keV}$, $H C=15-30 \mathrm{kev} / 10-15 \mathrm{keV}$. Symbols are as follows: rise0 (open black circles, only in KS 1947+300), rise1 (red circles), rise2 (orange squares), rise3 (pink crosses, only in KS 1947+300), peak (black triangle-up), decay1 (violet stars), decay2 (brown diamonds), decay3 (green triangle-down) and decay4 (blue pluses). See the electronic edition of the Journal for a colour version of this figure.

$(=0.001 \mathrm{~Hz} \approx 1 / T)$. Tables $3-5$ give the results of the power spectral analysis. Figures 6-9 show the power spectra and noise components of various spectral regions. Any given power spectrum can be fitted with the sum of two to four broad Lorentzian profiles (excluding the peaks), although we identify seven different types of noise components.

\subsection{Energy spectra}

The main objective of this work is to investigate the aperiodic variability of high-mass X-ray pulsars in correlation with the spectral states as defined in CD/HIDs, in the same way as it has been extensively done in low-mass X-ray binaries. A detailed spectral analysis is out of the scope of this work and will be performed in a future paper. The reader is referred to Wilson et al. (2008) (EXO 2030+375), Mowlavi et al. (2006) (V0332+53), Nakajima et al. (2006) (4U 0115+63) and Naik et al. (2006) (KS $1947+300)$ for a detailed analysis of the energy spectra during type II outbursts. However, for the sake of clarity in the discussion, it is illustrative to know the X-ray luminosity of the different spectral regions. Thus we obtained a time-averaged spectrum for each spectral region. The $3-30 \mathrm{keV}$ spectrum was fitted with an absorbed power-law model modified at high energies by an exponential cutoff. In addition, a Gaussian profile at $\sim 6.4 \mathrm{keV}$, that accounts for iron emission line, was required in most cases. The X-ray flux and luminosity in the $3-30 \mathrm{keV}$ energy range is given in Table 2. The distance to the sources is given in Table 1.

\section{Results}

\subsection{Outburst profiles}

The total duration of the $4 \mathrm{U} 0115+63$ outburst was $\sim 55$ days ( $\sim$ MJD 53 245-53 300) but the rise was shorter ( 11 days) than the decay $(\sim 27$ days). The brightest phase of the outburst displays a multi-peak profile and lasted for at least 15 days. The source spent about one third of the time in this bright state. The maximum flux was achieved on $\sim$ MJD53264 and amounted to $\sim 280 \mathrm{mCrab}$. The PCA observations of 4U 0115+63 began at the mid point of the rising part of the outburst on September 6, 2004 (MJD 53254) and finished on October 25, 2004 (MJD 53 304).

The ASM light curves of KS 1947+300 and EXO 2030+375 show very similar characteristics, despite the difference in overall luminosity (the EXO 2030+375 outburst was a factor of 4 brighter). Not only the outburst profile and duration $(\sim 165$ days and $\sim 155$ days, respectively) are similar but also the pre- and post-outburst X-ray activity. In these two sources the main outburst is preceded by a minor one, which started one orbital period before the onset of the major event. Likewise, the major outburst was followed by a series of smaller outbursts separated by a time interval consistent again with the orbital period (40 days in KS 1947+300 and 46 days in EXO 2030+375). The outburst profiles are quite symmetric with the rise and decay lasting for about 60-70 days. The KS 1947+300 ASM light curve contains a gap during the rise due to its proximity to the Sun at that time. 

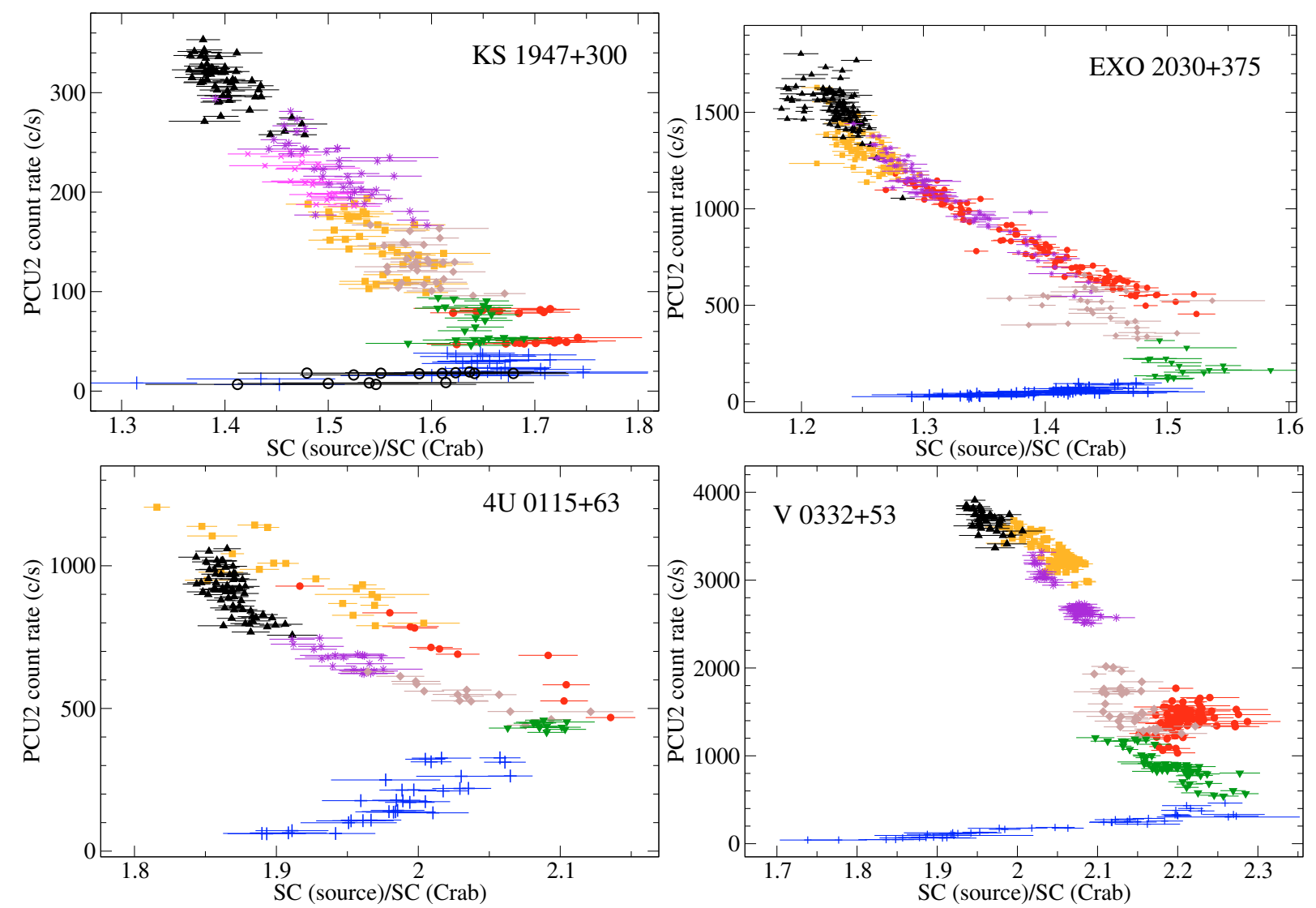

Fig. 4. Hardness-intensity diagrams. Each point represents a 512-s integration. Intensity corresponds to the energy range 4-30 keV. Symbols are as follows: rise0 (open black circles, only in KS 1947+300), rise1 (red circles), rise2 (orange squares), rise3 (pink crosses, only in KS 1947+300), peak (black triangle-up), decay1 (violet stars), decay2 (brown diamonds), decay3 (green triangle-down) and decay4 (blue pluses). See the electronic edition of the Journal for a colour version of this figure.

V0332+53 showed the most intense outburst. The maximum flux recorded in the energy range $1.3-12.1 \mathrm{keV}$ reached $\sim 1.5 \mathrm{Crab}$. The outburst began in 2004 November (MJD 53 330) and reached maximum flux about one month later ( MJD 53 368). The total duration of the outburst was $\sim 105$ days. As in $4 \mathrm{U} 0115+63$, the decay was slower than the rise. The difference appeared at the end of the decay, where a longer tail can be seen (Fig. 1).

There appears to be a relationship between the shape and duration of the outbursts and the orbital period of the system. KS 1947+300 and EXO 2030+375 have the wider orbits and show the longer and more symmetric outbursts. The outburst profiles of $4 \mathrm{U} 0115+63$ and $\mathrm{V} 0332+53$ have positive skewed (elongated tail at the right) and last shorter. The skewness statistics (the third moment about the mean normalised to the standard deviation, $\left.\frac{1}{N} \sum\left(x_{i}-\bar{x}\right)^{3} / \sigma^{3}\right)$ gives the following values for $4 \mathrm{U} 0115+63$, V0332+53, KS 1947+300 and EXO 2030+375: $0.24,0.58,0.16$ and -0.035 , respectively. In Be/X-ray binaries, the reservoir of matter available for accretion onto the neutron star companion comes from the circumstellar disc around the $\mathrm{Be}$ star's equator. The duration of the X-ray outbursts is expected to be proportional to the amount of accreted material. Thus longer outburst duration implies larger discs. This result agrees with the correlation between the orbital period and strength of the H $\alpha$ line (Reig et al. 1997; Reig 2007). The longer the orbital period, the wider the orbit, the larger the disc. This correlation is explained by the truncation of the disc by the neutron star, which will be more efficient for narrower orbits. Okazaki \& Negueruela (2001) estimated that the Be discs in 4U 0115+63,
V0332+53 and EXO $2030+375$ are truncated at the $4: 1$ resonance radius, while that of KS $1947+300$ at the 3:1 resonance. In terms of stellar radii, assuming the stellar parameters given in Okazaki \& Negueruela (2001), these resonance radii translate into truncation radii $\sim 4.7 R_{*}, \sim 5.6 R_{*}, \sim 6.9 R_{*}$ and $\sim 8 R_{*}$ for $4 \mathrm{U} 0115+63$, V0332+53, EXO 2030+375 and KS 1947+300, respectively. This numbers should be taken with caution since the calculations are strongly dependent on the disc viscosity and assume the same disc physical parameters in all systems.

\subsection{Colour analysis}

Be/X-ray binaries show two spectral branches in the CD/HID. We shall refer to these branches as the low-intensity or horizontal branch and the high-intensity or diagonal branch. However, not all sources display the entire pattern of variability. Sometimes only one branch is visible in the CD. In KS 1947+300, the scatter of the points that populate the low-intensity branch is too large to clearly define the branch (see Fig. 3). In EXO 2030+375, only the hardest (in terms of SC) part of this branch is visible.

Unless the motion in the horizontal branch is faster than $\sim 1$ day $^{-1}$, it seems unlikely that the lack of a complete branch in KS $1947+300$ and EXO 2030+375, at the end of the outburst, is due to observational gaps. At least in EXO 2030+375, the frequency of the PCA observations was $\sim 1$ day $^{-1}$. More likely, the lack of a clear horizontal branch in the $\mathrm{CD}$ of these two sources can be attributed to the fact that they did not return to quiescence. Both KS 1947+300 and EXO 2030+375 showed smaller outbursts (type I) after the major outburst (type II) analysed in this 
Table 3. Power spectral parameters (centroid frequency, $F W H M$ and rms) for the $L_{\mathrm{b}}, L_{\mathrm{l}}$ and $L_{\mathrm{u}}$ components. Errors are $90 \%$ confidence level. The centroid frequency of the zero-centred Lorentzian was fixed during the fit. HB stands for horizontal branch.

\begin{tabular}{|c|c|c|c|c|c|c|c|c|c|c|c|}
\hline $\begin{array}{l}\text { Spectral } \\
\text { region }\end{array}$ & $\begin{array}{r}L_{\mathrm{x}} \\
\times 10^{37} \\
\mathrm{erg} \mathrm{s}^{-1} \\
\end{array}$ & $\nu_{\mathrm{b}}$ & $\begin{array}{r}L_{\mathrm{b}} \\
F W H M_{\mathrm{b}} \\
10^{-3}(\mathrm{~Hz}) \\
\end{array}$ & $\begin{array}{c}\mathrm{rms}_{\mathrm{b}} \\
(\%)\end{array}$ & $\begin{array}{c}v_{1} \\
(\mathrm{~Hz})\end{array}$ & $\begin{array}{c}L_{\mathrm{l}} \\
F W H M_{1} \\
(\mathrm{~Hz})\end{array}$ & $\begin{array}{c}\mathrm{rms}_{1} \\
(\%)\end{array}$ & $\begin{array}{c}v_{\mathrm{u}} \\
(\mathrm{Hz})\end{array}$ & $\begin{array}{c}L_{\mathrm{u}} \\
F W H M_{\mathrm{u}} \\
(\mathrm{Hz}) \\
\end{array}$ & $\begin{array}{c}\mathrm{rms}_{\mathrm{u}} \\
(\%)\end{array}$ & $\begin{array}{l}\chi^{2} / \\
\text { dof* }\end{array}$ \\
\hline \multicolumn{12}{|c|}{$4 \mathrm{U} 0115+63$} \\
\hline rise 1 & 7.5 & 0 & $2.0^{\text {fixed }}$ & $19 \pm 3$ & 0 & $1.6 \pm 0.3$ & $8.7 \pm 0.6$ & - & - & - & $1.4 / 78$ \\
\hline rise 2 & 11.8 & 0 & $2.0^{\text {fixed }}$ & $12 \pm 2$ & 0 & $0.87 \pm 0.06$ & .2 & $0.1 \pm 0.1$ & $4.1 \pm 0.3$ & $.2 \pm 0.3$ & $1.3 / 76$ \\
\hline peak & 11.0 & $* *$ & ** & ** & 0 & $0.78 \pm 0.08$ & $5 \pm$ & $0.4 \pm 0.3$ & $4.9 \pm 0.7$ & $.0 \pm 0.7$ & $2.0 / 76$ \\
\hline deca & 7.9 & $* *$ & $* *$ & $* *$ & 0 & $0.72 \pm 0.07$ & $10.0 \pm 0.3$ & $1.5 \pm 0.7$ & $4.3 \pm 0.9$ & $.9 \pm 0.4$ & $1.3 / 76$ \\
\hline decay2 & 6.0 & 0 & $3.0 \pm 2.0$ & $13 \pm 3$ & 0 & $0.76 \pm 0.10$ & $9.7 \pm$ & 0 & $5.3 \pm 1.0$ & $.7 \pm 0.7$ & $1.9 / 74$ \\
\hline deca & 5.0 & $* *$ & F* & $* *$ & 0 & $0.73 \pm 0.04$ & 11 & - & - & - & $1.3 / 75$ \\
\hline deca & 0.9 & - & - & - & $10 \pm 0.01$ & $0.34 \pm 0.01$ & 20 & - & - & - & $1.1 / 78$ \\
\hline \multicolumn{12}{|c|}{ KS $1947+300$} \\
\hline rise 0 & 0.1 & - & - & - & 0 & $0.087 \pm 0.005$ & .7 & - & - & - & $2.1 / 83$ \\
\hline rise 1 & 0.8 & - & - & - & 0 & $0.14 \pm 0.01$ & 17.0 & - & - & - & $1.6 / 86$ \\
\hline rise2 & 2.3 & 0 & $9 \pm 3$ & $9.7 \pm 0.5$ & 0 & $0.40 \pm 0.04$ & 14. & 0 & $2.3 \pm 0.8$ & $.3 \pm 1.2$ & $0.9 / 82$ \\
\hline rise 3 & 4.3 & 0 & $15 \pm 10$ & $8.7 \pm 0.9$ & 0 & $0.57 \pm 0.04$ & $3.2 \pm 0.2$ & 0 & $4.0 \pm 0.8$ & $2 \pm 0.4$ & $1.7 / 82$ \\
\hline $\mathrm{p}$ & 6.3 & 0 & $12 \pm 2$ & $9.5 \pm 0.5$ & 0 & $0.65 \pm 0.04$ & $14.3 \pm 0.2$ & $.1 \pm 0.3$ & $3.6 \pm 0.2$ & $.5 \pm 0.5$ & $1.3 / 81$ \\
\hline $\mathrm{d}$ & 4.7 & 0 & $9 \pm 2$ & $10.2 \pm 0.7$ & 0 & $48 \pm 0.05$ & 13. & 0 & $2.7 \pm 0.4$ & $7.7 \pm 0.6$ & $1.0 / 82$ \\
\hline & 2.6 & 0 & $14 \pm 3$ & $9.2 \pm 0.8$ & 0 & $61 \pm 0.04$ & 4 & - & - & - & $1.5 / 84$ \\
\hline & 1.3 & 0 & $11 \pm 9$ & $4 \pm 0$ & 0 & $52 \pm 0$ & & - & - & - & $1.0 / 84$ \\
\hline decas & 0.01 & - & - & - & 0 & $0.10 \pm 0.02$ & & - & - & - & $1.4 / 87$ \\
\hline \multicolumn{12}{|c|}{ EXO 2030+375 } \\
\hline rise 1 & 8.4 & 0 & $44 \pm 6$ & $15.2 \pm 0.5$ & 0 & $0.50 \pm 0.02$ & 21 & 0 & $2.9 \pm 0.2$ & $10.5 \pm 0.2$ & $1.7 / 81$ \\
\hline rise2 & 12.7 & 0 & \pm 6 & $9 \pm 0.4$ & $0.07 \pm 0$ & \pm 0.02 & 0.2 & $9 \pm 0.15$ & \pm 0.1 & $8 \pm 0.7$ & $1.8 / 79$ \\
\hline & 14.5 & 0 & $3.3 \pm 2.0$ & $7 \pm 2.1$ & $0 \pm$ & $0.50 \pm 0.02$ & & $0.79 \pm($ & $2.4 \pm 0.2$ & $12.3 \pm 1.4$ & $2.5 / 75$ \\
\hline & 9.7 & 0 & $3.4 \pm 1.0$ & $10.2 \pm 1.0$ & $0.17 \pm 0$ & $0.64 \pm 0.09$ & 22. & $0.74 \pm 0.60$ & $2.3 \pm 0.8$ & $10.4 \pm 4.9$ & $1.4 / 76$ \\
\hline ? & 3.4 & 0 & $12 \pm 5$ & $8.1 \pm 0.9$ & $0.11 \pm 0.04$ & $0.83 \pm 0.07$ & 21.7 & $0.54 \pm 0.60$ & $5.3 \pm 1.2$ & $9.2 \pm 0.9$ & $1.3 / 80$ \\
\hline decav 3 & 0.7 & 0 & $42 \pm 20$ & $9.2 \pm 0.8$ & $0.20 \pm 0.09$ & $0.64 \pm 0.0$ & $15.6 \pm 0.7$ & $0.95 \pm 0.80$ & $4.3 \pm 0.8$ & $7.8 \pm 0.4$ & $1.1 / 78$ \\
\hline deca & 0.4 & - & - & - & 0 & $0.046 \pm 0.005$ & $22.1 \pm 0.9$ & - & - & - & $2.0 / 85$ \\
\hline \multicolumn{12}{|c|}{ V0332+53 } \\
\hline rise 1 & 13.5 & 0 & \pm 0.4 & \pm( & $<0.007$ & $0.70 \pm 0.02$ & & - & - & - & $2.1 / 80$ \\
\hline ri & 29.3 & 0 & $7.0 \pm 2.5$ & $3.5 \pm 0.3$ & $0.33 \pm 0.04$ & $1.54 \pm 0.06$ & $7.6 \pm$ & - & - & - & $1.5 / 79$ \\
\hline peak & 32.2 & 0 & $7.6 \pm 4.0$ & $3.8 \pm 0.5$ & $0.8 \pm 0.1$ & $2.0 \pm 0.09$ & $6.0 \pm 0.3$ & - & - & - & $2.5 / 76$ \\
\hline decay1 & 24.6 & 0 & $3.7 \pm 2.0$ & $3.1 \pm 0.3$ & $0.04 \pm 0.02$ & $0.85 \pm 0.05$ & $8.6 \pm 0.1$ & - & - & - & $1.2 / 80$ \\
\hline decay2 & 15.2 & 0 & $2.5 \pm 0.3$ & $6.8 \pm 1.1$ & $0.08 \pm 0.01$ & $0.86 \pm 0.01$ & $10.2 \pm 0.2$ & - & - & - & $2.3 / 81$ \\
\hline decay3 & 8.8 & 0 & $1.9 \pm 0.9$ & $8.1 \pm 0.6$ & $0.04 \pm 0.01$ & $0.54 \pm 0.01$ & $13.1 \pm 0.3$ & - & - & - & $1.3 / 81$ \\
\hline decay4(HB) & 1.7 & 0 & $7.2 \pm 4.0$ & $9.5 \pm 1.1$ & $0.09 \pm 0.01$ & $0.21 \pm 0.01$ & $20.9 \pm 0.3$ & - & - & - & $1.5 / 85$ \\
\hline
\end{tabular}

*: Including components in Tables 4 and 5 .

**: Affected by the mHz QPO. See Fig. 5 and Table 5. See also Coburn et al. (2004).

Table 4. Power spectral parameters (centroid frequency, $F W H M$ and rms) for the $L_{\mathrm{u}^{\prime}}$ component in EXO 2030+375. Errors are $90 \%$ confidence level.

\begin{tabular}{lccc}
\hline \hline $\begin{array}{l}\text { Spectral } \\
\text { region }\end{array}$ & $\begin{array}{c}v_{\mathrm{u}^{\prime}} \\
(\mathrm{Hz})\end{array}$ & $\begin{array}{c}F W H M_{\mathrm{u}^{\prime}} \\
(\mathrm{Hz})\end{array}$ & $\begin{array}{c}\mathrm{rms}_{\mathrm{u}^{\prime}} \\
(\%)\end{array}$ \\
\hline rise1 & - & - & - \\
rise2 & $2.5 \pm 0.1$ & $5.7 \pm 0.2$ & $7.2 \pm 0.3$ \\
peak & $3.1 \pm 0.6$ & $6.6 \pm 0.4$ & $7.1 \pm 1.0$ \\
decay1 & $2.3_{-0.4}^{+1.1}$ & $6.7 \pm 0.7$ & $7.8 \pm 2.2$ \\
decay2 & - & - & - \\
decay3 & - & - & - \\
decay4 & - & - & - \\
\hline
\end{tabular}

work. The X-ray emission level did not return to zero in these two systems (i.e. below the sensitivity threshold of the PCA) as can be seen in Fig. 2. The average count rate of the last observations was $30 \mathrm{c} \mathrm{s}^{-1} \mathrm{PCU}^{-1}$ in EXO 2030+375 and $5 \mathrm{c} \mathrm{s}^{-1} \mathrm{PCU}^{-1}$ in $\mathrm{KS} 1947+300$. In contrast, $4 \mathrm{U} 0115+63$ seems to have gone under the detectability limit of the instruments. It is reasonable to think that if the intensity had gone lower, then the horizontal branch in KS $1947+300$ and EXO 2030+375 would have shown up more clearly. Note that V0332+53 also went back to $\sim 0 \mathrm{c} \mathrm{s}^{-1}$ and also displays two distinct branches in the CD.

In LMXBs, the patterns observed in a $\mathrm{CD}$ are often also recognisable in the corresponding HID. Sometimes, however, spectral branches are more distinct in one of the two diagrams. Whether CD or HID presents the cleanest pattern depends on source and on the quality of the data. Figure 4 displays the HID of the four X-ray pulsars analysed in this work. In contrast to the CD, the two branches are clearly seen for all four sources when the $\mathrm{HC}$ is replaced by the intensity. The junction of the two branches occur when the count rate is at $\sim 10 \%$ of the peak value $(\sim 30 \%$ in $4 \mathrm{U} 0115+63)$. The second column in Table 2 indicates the branch in which the source lay during those particular observations. For practical reasons, the name "horizontal branch" is also used if intensity is plotted on a logarithmic scale in the HID, even though in this case this branch does not appear horizontal.

The low-intensity (horizontal) branch is populated by points with a low count rate. Since the PCA monitoring began when the outbursts were already in progress, the low-intensity branch contains points from the end of the outbursts. The only source for which data prior to the onset of the outburst are available is 
KS 1947+300. As can be seen in Fig. 4, the data points (open black circles) that corresponds to the very beginning of the outburst also lie in the low-intensity branch. Thus we conclude that the horizontal branch represents the start and end point of the source in its journey through the CD/HID. In terms of X-ray luminosity, this branch appears when $L_{\mathrm{X}} \lesssim 10^{37} \mathrm{erg} \mathrm{s}^{-1}$.

The evolution of the X-ray colours as the outburst evolves, particularly the hard colour (HC), clearly distinguishes V0332+53 from the other three systems. Unlike, KS 1947+300, 4U 0115+63 and EXO 2030+375, both the SC and HC in $\mathrm{V} 0332+53$ decrease as the count rate increases (see Fig.2). This different behaviour of the $\mathrm{HC}$ implies that the motion in the diagonal branch, as the count rate increases, is from the bottom right part of the CD to top left part in 4U $0115+63$, KS 1947+300 and EXO 2030+375 and from bottom left to top right in V0332+53. Another important difference is that the low-intensity branch is harder than the high-intensity branch in V0332+53, while it is softer in EXO 2030+375, KS 1947+300 and 4U 0115+63 (Fig. 3). In this latter case, the horizontal branch corresponds to a low/soft (in terms of the hard colour) state that it is not seen in low-mass X-ray binaries or black-hole systems.

After the peak, the source returns following the reverse track. The sources spend most of the outburst in the diagonal branch. The sources stay in this branch about $70 \%-80 \%$ of the duration of the outburst. As can be inferred from Table 2, typical timescales are months in the diagonal branch and weeks in the horizontal branch.

$4 \mathrm{U} 0115+63$ exhibits hysteresis, that is, the X-ray colours adopt different values depending on whether the source is on the rise or the decay of the outburst, despite that the count rate is similar. This effect is also present in V0332+53 and EXO $2030+375$, although not as significant as in $4 \mathrm{U} 0115+63$. It is absent in KS 1947+300 (see Fig. 4). In EXO 2030+375 and $\mathrm{V} 0332+53$ both X-ray colours are larger during the rise than during the decay, that is, the sources present a harder spectrum during the rise. In $4 \mathrm{U} 0115+63$, the changes are more complex. During the rise SC is larger and $\mathrm{HC}$ is smaller than during the decay. Although the larger error bars in KS 1947+300 might prevent us from detecting hysteresis in this source, an effect with the amplitude seen in $4 \mathrm{U} 0115+63$ should also be seen in the SC-intensity diagram of KS 1947+300.

Interestingly, cyclotron resonance scattering features have been observed for all sources except KS 1947+300. In particular, $4 \mathrm{U} 0115+63$ shows up to five cyclotron line harmonics (Coburn et al. 2004). However, while the energy of the fundamental line is found at $11 \mathrm{keV}$ in $4 \mathrm{U} 0115+63$ (Santangelo et al. 1999), it appears at $27 \mathrm{keV}$ in $\mathrm{V} 0332+53$ (Pottschmidt et al. 2005). The cyclotron line in EXO 2030+375, at $11 \mathrm{keV}$, is less significant and lacks harmonic content (Wilson et al. 2008). According to the definition of the X-ray colours given in Sect. 4.2, the HC in $4 \mathrm{U} 0115+63$ is expected to be more strongly affected by the presence of the cyclotron line and its harmonics, while in V0332+53 and EXO 2030+375 its contribution to the variability in $\mathrm{HC}$ would be insignificant. Moreover, the energy of the fundamental cyclotron line in $4 \mathrm{U} 0115+63$ has been seen to increase from $\sim 11 \mathrm{kev}$ to $16 \mathrm{keV}$ as the X-ray luminosity decreased below $5 \times 10^{37} \mathrm{erg} \mathrm{s}^{-1}$ (Nakajima et al. 2006). The relationship between hysteresis, as seen in the HID, and cyclotron lines needs to be verified through detailed spectral analysis.

\subsection{Aperiodic variability}

The power spectra of Be/X-ray binaries is dominated by the peaks of the pulse component and its harmonics. In order to

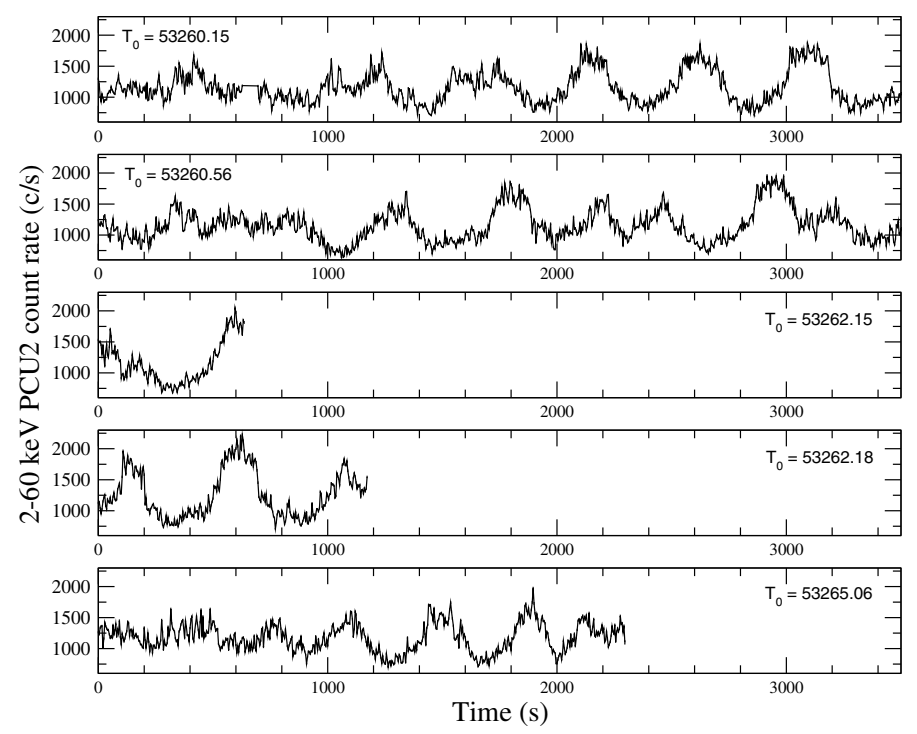

Fig. 5. Quasi-periodic oscillations near the peak of the outburst in $4 \mathrm{U} 0115+63$. Time zero is indicated in MJD in each panel. The time resolution is $4 \mathrm{~s}$.

study the aperiodic variability (band-limited noise and possible QPOs), the pulse noise should be removed. However, this is not an easy task. Some authors substituted the points contaminated by the pulses and their harmonics by a spline fit to the remaining points or to a power law connecting the average of a few points before and after the pulse gap (Belloni \& Hasinger 1990). This procedure does not give satisfactory results if the peaks are too broad. Others simply remove the points from the power spectra (Reig et al. 2006). Angelini et al. (1989) removed the pulse noise after fitting it with a model consisting of the first 10 harmonics of the coherent pulsar signal and a power law. In this work we have opted to maintain the peaks and fit them with Lorentzians. In addition to the distortion introduced by the pulse noise, the power spectra continuum of Be/X-ray binaries contain significant substructures only visible in certain states. Sometime the continuum shows bumps and wiggles, making it difficult to obtain good fits. This difficulty was also found by Belloni \& Hasinger (1990), who could not fit the power spectra of a number of high-mass X-ray binaries with simple analytical laws, such as power law and cutoff, as in low-mass X-ray binaries.

In general, the power spectra of $\mathrm{Be} / \mathrm{X}$-ray binaries is characterised by band-limited noise (BLN), that is, a steeping of the power density toward high frequency and a flattening toward low frequency. Although a double broken power law may give good fits in some cases (KS 1947+300) it cannot fit all sources and all states (EXO 2030+375). In addition, all sources present peaked noise in certain spectral states.

Despite this complexity, we find that the power spectra of $\mathrm{Be} / \mathrm{X}$-ray binaries can be fitted acceptably with the sum of a small number of Lorentzian functions. Ignoring the peaks of the pulse component, 2-4 Lorentzian profiles are needed to obtain acceptable fits. However, we identify 7 different types of noise components. 4 of these components are band-limited (BLN) and 3 QPO. The results of the power spectral fitting are given in Tables 3-5. Figures 6-9 show the power spectra of the four $\mathrm{X}$-ray pulsars for various regions. Different line types indicate different noise components as explained below.

$L_{\mathrm{b}}$ (dotted line) is a zero-centred Lorentzian that describes the noise below $0.01 \mathrm{~Hz}$. Due to the very low characteristic frequency of this component (often peaks outside the available 

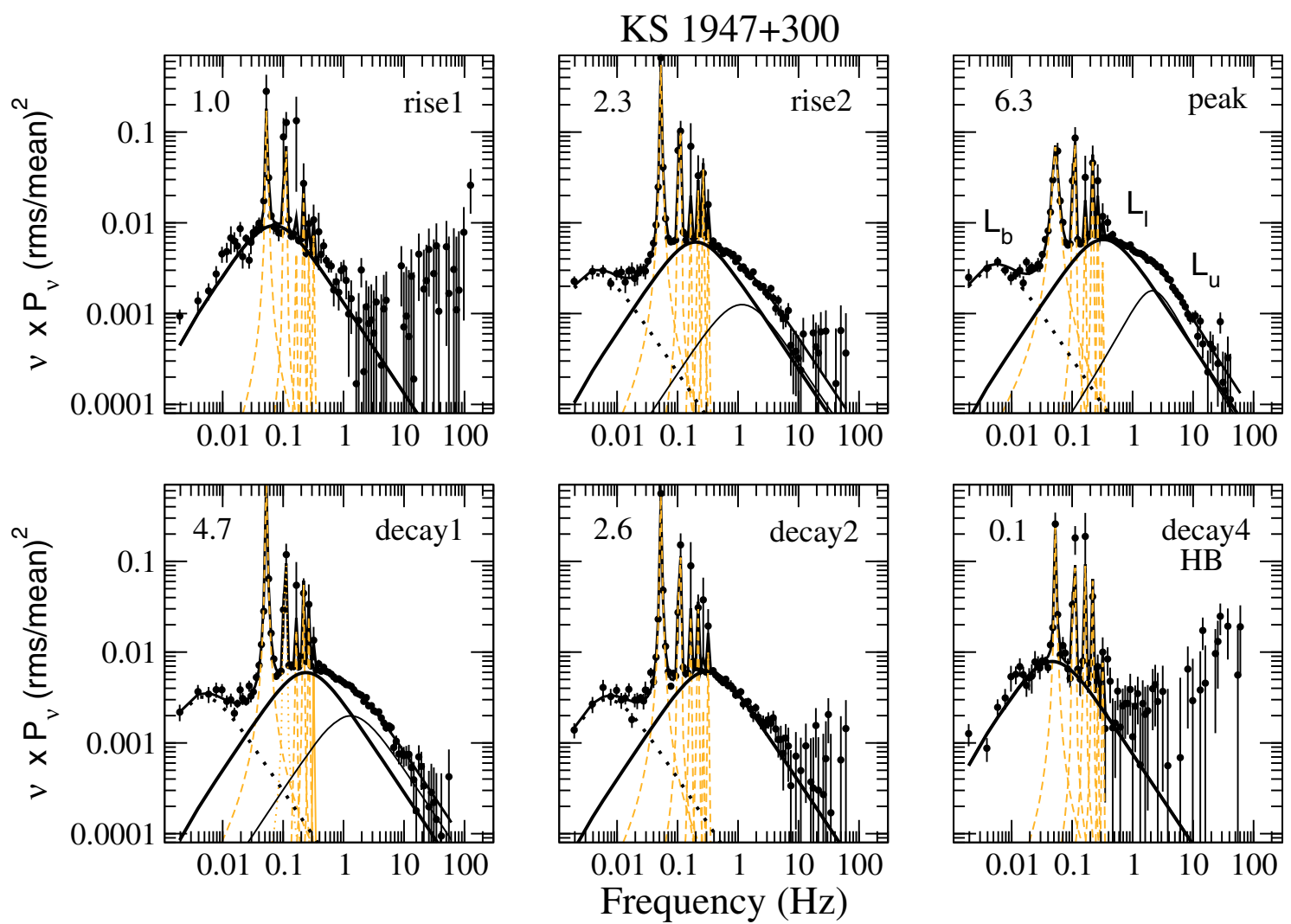

Fig. 6. Power spectra of KS $1947+300$ and fit functions of various spectral regions. The number at the top left of each panel is the X-ray luminosity in units of $10^{37} \mathrm{erg} \mathrm{s}^{-1}$. HB stands for horizontal branch. The different lines indicate the individual Lorentzian components of the fit: $L_{\mathrm{b}}$ (dotted), $L_{\mathrm{l}}$ (thick solid black), $L_{\mathrm{u}}$ (thin solid black). The pulse peaks (fundamental and harmonics) are represented by thin dashed orange lines.

frequency range) it is not always well constrained. In some states of $4 \mathrm{U} 0115+63, L_{\mathrm{b}}$ is modified by the appearance of a slow QPO. $L_{\mathrm{b}}$ disappears in the horizontal branch of all sources except in V0332+53.

$L_{1}$ (thick solid line) is also a broad component with $Q<0.4$ or centred at zero. Its characteristic frequency shifts to low frequencies when the count rate is low. This component dominates most of the power spectrum, covering the range from $\sim 0.1-$ $10 \mathrm{~Hz}$. In some cases (V0332+53), it is the only component fitting the high-frequency end of the power spectrum.

In $4 \mathrm{U} 0115+63$, KS $1947+300$ and EXO 2030+375 one or two more Lorentzians are needed to account for the highfrequency noise. We shall call these component $L_{\mathrm{u}}$ (black thin solid line) and $L_{\mathrm{u}^{\prime}}$ (grey thick solid line), with characteristic frequencies $v_{\mathrm{u}} \approx 1-3 \mathrm{~Hz}, v_{\mathrm{u}^{\prime}} \approx 4 \mathrm{~Hz}$, respectively and $Q \approx 0-0.5$. These components only appear when the count rate is high, i.e., near and at the peak the outburst.

In general, $L_{\mathrm{b}}$ accounts for the noise short-ward of the main pulse peak, while $L_{1}$ and $L_{\mathrm{u}} / L_{\mathrm{u}^{\prime}}$ fit the power spectrum above the spin frequency. Together they account for the band-limited noise.

The faster pulsars (V0332+53 and 4U 0115+63) display peaked noise components, which we shall refer to as QPO noise, even though the $Q$ value is not always larger than 2 . Table 5 gives the best-fit parameters of these components. $L_{\mathrm{LF}}$ (dashed line in Figs. 8 and 9) is common to the two sources and has a characteristic frequency $v_{\mathrm{LF}} \approx 0.05 \mathrm{~Hz}$ and $0.5 \gtrsim Q_{\mathrm{LF}} \gtrsim 2$. It becomes stronger as the count rate decreases. Note that although the centroid frequency of this Lorentzian component increases as the count rate decreases, its width decreases. The characteristic frequency (i.e. maximum frequency) remains fairly constant at the value indicated above $(\sim 0.05 \mathrm{~Hz}) . \mathrm{V} 0332+53$ shows, in addition, another QPO (that we shall call $L_{\mathrm{s}}$, dotted-dashed line) whose centroid frequency coincides with the frequency of the fundamental peak $(0.23 \mathrm{~Hz})$ of the pulse period and remains constant throughout the outburst. The strength of $L_{\mathrm{s}}$ increases with count rate, reaching maximum during the peak of the outburst. However, it disappears in low-intensity states, when $L_{X}$ goes below $\sim 10^{37} \mathrm{erg} \mathrm{s}^{-1}$. 4U 0115+63 also shows another QPO at $\sim 3 \mathrm{mHz}$. This QPO is even visible in the light curve (Fig. 5) and has also been reported in previous outbursts of the source (Heindl et al. 1999; Coburn et al. 2004). We have called this component $L_{\mathrm{mQPO}}$.

\section{Discussion}

In this section, we compare the correlated spectral and timing variability of high-mass X-ray binaries (HMXBs) and low-mass $\mathrm{X}$-ray binaries (LMXBs). We comment on the similarities and differences of these two types of X-ray binaries.

\subsection{Comparison with low-mass $X$-ray binaries}

The Be/X-ray binaries that display a wide dynamic range in intensity (type II outbursts) trace two-branch patterns in their CD/HID. As in low-mass systems, the sources do not jump through the diagram but moves smoothly, following the pattern. The horizontal branch (HB) corresponds to a low-intensity state and shows the higher fractional rms, similar to the the island state in atolls and horizontal branch in $\mathrm{Z}$ sources. This branch represents the start and end states of the source through the outburst. In the high-intensity diagonal branch (DB), the noise 

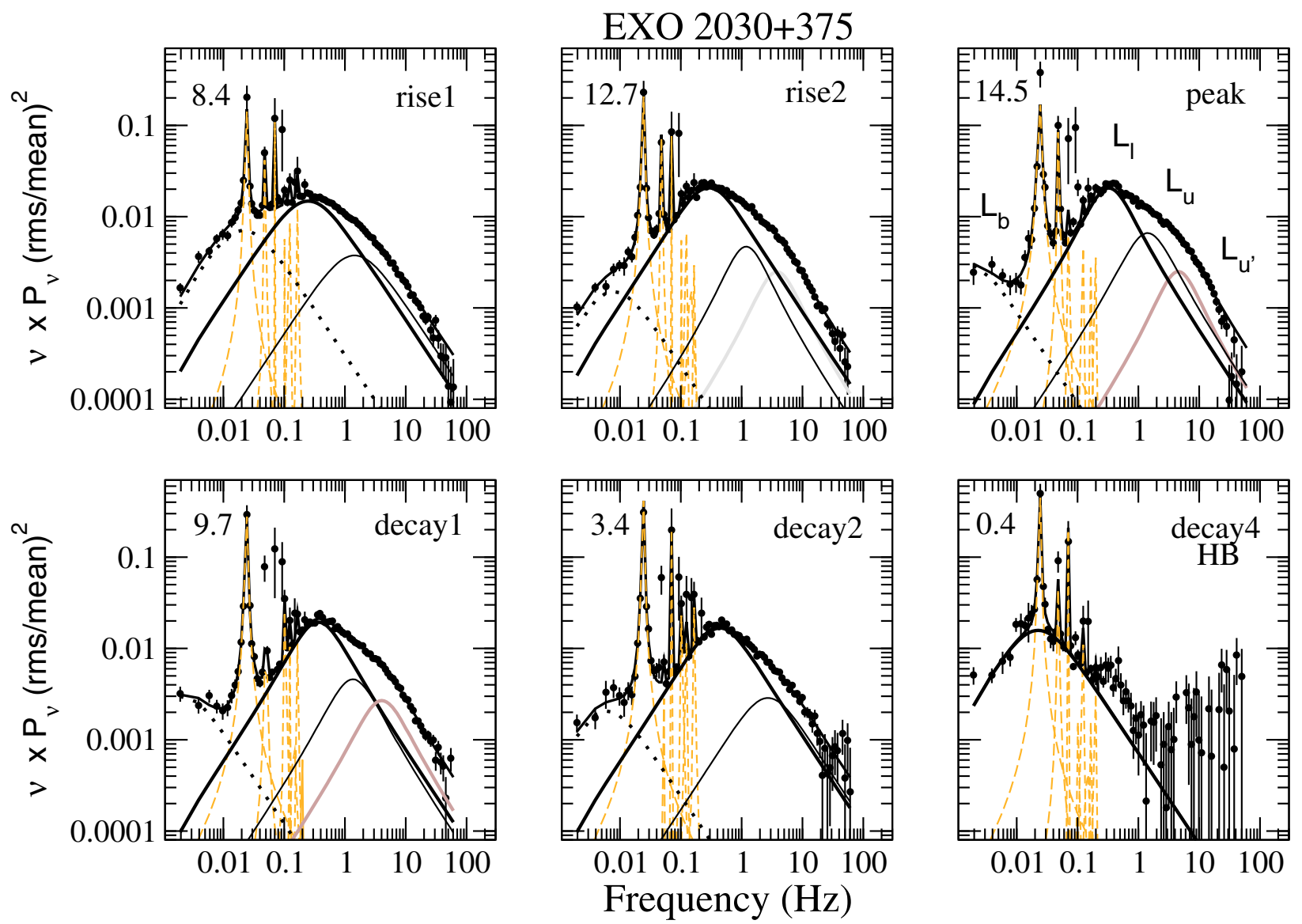

Fig. 7. Power spectra of EXO $2030+375$ and fit functions of various spectral regions. The number at the top left of each panel is the X-ray luminosity in units of $10^{37} \mathrm{erg} \mathrm{s}^{-1}$. HB stands for horizontal branch. The different lines indicate the individual Lorentzian components of the fit: $L_{\mathrm{b}}$ (dotted), $L_{\mathrm{l}}$ (thick solid black), $L_{\mathrm{u}}$ (thin solid black), $L_{\mathrm{u}^{\prime}}$ (thick solid grey). The pulse peaks (fundamental and harmonics) are represented by thin dashed orange lines.

components display higher characteristic frequencies and lower rms than in the HB.

The power spectra of LMXBs in the frequency range below $100 \mathrm{~Hz}$ are characterised by (Belloni et al. 2002; van Straaten et al. 2002, 2003; Olive et al. 2003; van der Klis 2006): i) a power-law red noise component in the lowest frequency range, traditionally known as very-low frequency noise (VLFN). This component is weak or absent in the island and horizontal branches of atoll $/ Z$ sources and increases in strength as the source moves along the branches; ii) strong band-limited noise (BLN), consisting of flat-topped noise that breaks at a frequency $v_{\mathrm{b}} \sim 0.01-50 \mathrm{~Hz}$. This component can be represented by a zero-centred Lorentzian and fits the low-frequency end of the power spectrum; iii) peaked noise at frequency $\sim 5 v_{\mathrm{b}}$. When this peaked noise appears as a QPO then it is called $L_{\mathrm{LF}}$ and its characteristic frequency $\nu_{\mathrm{LF}}$. Otherwise, it is normally referred as $L_{\mathrm{h}}$. Sometimes, both components are present, i.e., a narrow $L_{\mathrm{LF}}$ and a broad $L_{\mathrm{h}}$; iv) two zero-centred Lorentzians fitting the high-frequency part of the power spectrum. They normally appear when $v_{\mathrm{b}} \lesssim 1 \mathrm{~Hz}$ and are given the names $L_{\mathrm{l}}$ and $L_{\mathrm{u}}$, respectively. It is always observed that $v_{\mathrm{b}}<v_{\mathrm{LF}}<v_{1}<v_{\mathrm{u}}$.

In Be/X-ray binaries the power spectra can also be well fitted by a relatively small number of Lorentzian components. Some of these components can be identified in the power spectra of LMXBs. As in LMXBs, the band-limited noise in Be/X-ray binaries is fitted by three broad Lorentzians: $L_{\mathrm{b}}$ at low frequencies and $L_{1}$ and $L_{\mathrm{u}}$ at high frequencies. In Fig. 10 we have plotted the relationship between the characteristic frequencies, i.e. $v_{\max }=$ $\left(v_{0}^{2}+(F W H M / 2)^{2}\right)^{1 / 2}$, of $L_{\mathrm{b}}, L_{\mathrm{LF}, \mathrm{h}}$ and $L_{\mathrm{l}}$ for the HMXBs $4 \mathrm{U}$ $0115+63$ (filled squares) and V0332+53 (open squares) and for the LMXBs (Belloni et al. 2002; van Straaten et al. 2002, 2003; Reig et al. 2004) 4U 1728-34 (black filled circles), 4U 160852 (cyan filled triangles), GS 1826-24 (orange filled circles), 1E 1724-3045 (open circles), 4U 0614+09 (diamonds) and Aql X-1 (stars). For Aql X-1, $L_{\mathrm{h}}$ was plotted. They are known as the WK relation (after Wijnands \& van der Klis 1999a, upper panel) and the PBK relation (after Psaltis et al. 1999, lower panel). The fact that the data points corresponding to the Be/X-ray binaries fall on the global correlations found for LMXBs, if extrapolated at low frequencies, supports the identification of the noise components.

There are some important differences in the rapid aperiodic noise components between HMXBs and LMXBs, namely: i) the lower characteristic frequencies, ii) the lack of a clear correlation of the noise component parameters with mass accretion rate and iii) the noise component, $L_{\mathrm{s}}$, associated with the peaks of the periodic modulation.

In accretion-powered X-ray pulsars, QPOs fall in the milliHz range, which is a few orders of magnitude lower than in LMXBs. There are about 12 high-mass X-ray pulsars that show QPOs in their power spectra. The frequencies of these QPOs range from 1-400 mHz (Shirakawa \& Lai 2002; Inam et al. 2004; Mukherjee et al. 2006). Recently, Kaur et al. (2007) reported the discovery of a 1.3 Hz QPO in XTE J0111.2-7317, a Be/X-ray 

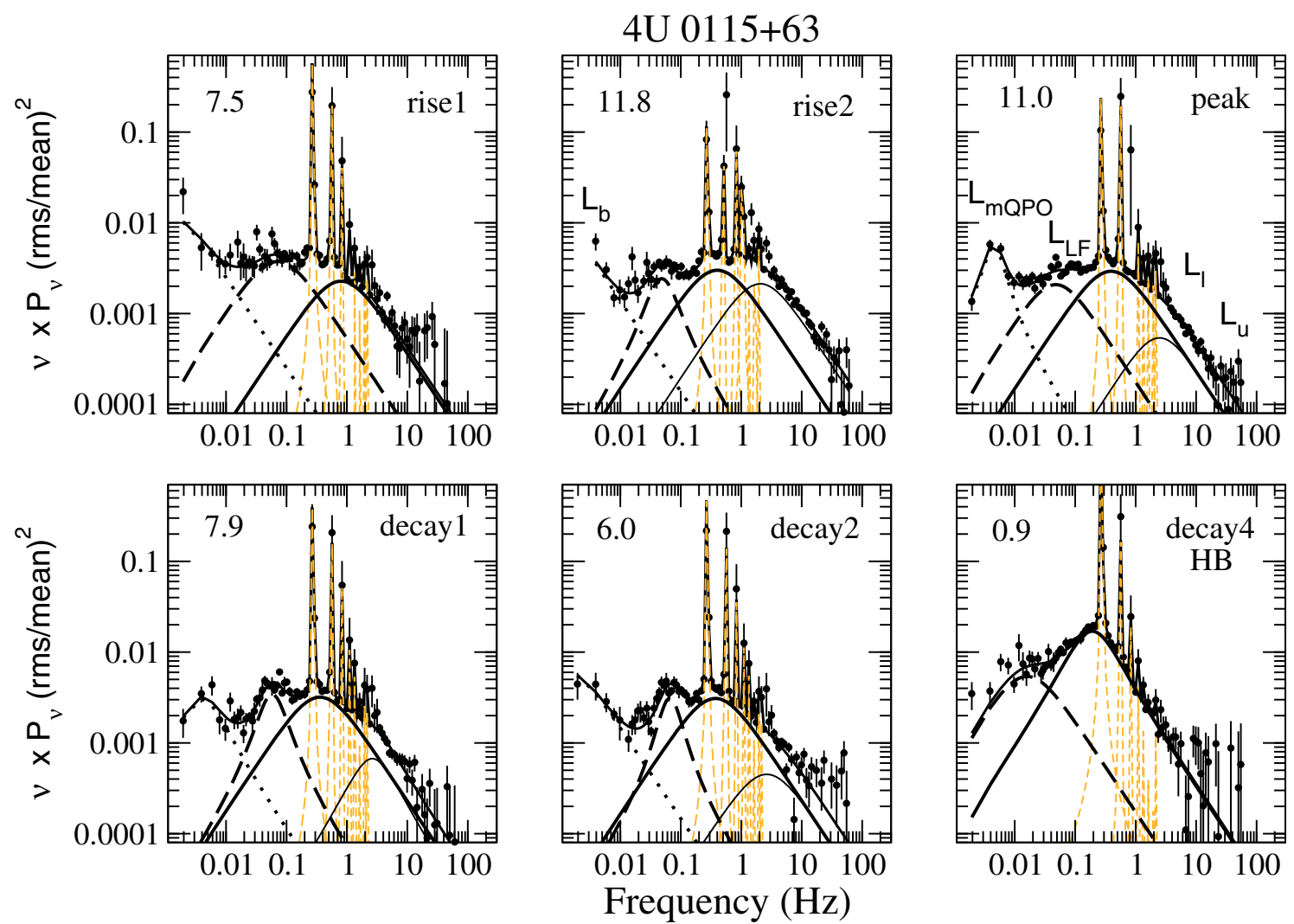

Fig. 8. Power spectra of $4 \mathrm{U} 0115+63$ and fit functions of various spectral regions. The number at the top left of each panel is the X-ray luminosity in units of $10^{37} \mathrm{erg} \mathrm{s}^{-1}$. HB stands for horizontal branch. The different lines indicate the individual Lorentzian components of the fit: $L_{\mathrm{b}}$ (dotted), $L_{\mathrm{l}}$ (thick solid black), $L_{\mathrm{LF}}$ (thick dashed), $L_{\mathrm{u}}$ (thin solid black). The pulse peaks (fundamental and harmonics) are represented by thin dashed orange lines. In "peak" and "decay1" the $L_{\mathrm{b}}$ component is affected by the $3 \mathrm{mHz} \mathrm{QPO}, L_{\mathrm{VLF}}$ (see text and Fig. 5).

binary located in the SMC. Many of these QPOs have $Q$ values lower than 2 , hence strictly speaking, they should be referred as peaked noise (conventionally, peaked noise is considered as a QPO when the quality factor $Q=v / F W H M>2$ ). However, since these features (also $L_{\mathrm{LF}}$ and $L_{\mathrm{s}}$ in this work) represent a substantial concentration of power in a limited frequency range they are referred to as QPOs.

If it is assumed that QPOs are formed as a result of Keplerian motion of inhomogeneities in an accretion disc then the longer implied timescales of the QPOs in HMXB is somehow expected as the inner radius of the accretion disc must be larger than the magnetospheric radius $\left(r_{\mathrm{m}} \sim 10^{8} \mathrm{~cm}\right) .10-400 \mathrm{mHz}$ QPOs imply disc radius of the order of $10^{8}-10^{9} \mathrm{~cm}\left(R_{\mathrm{d}} \sim\left(G M / 4 \pi^{2} v_{\mathrm{QPO}}^{2}\right)^{1 / 2}\right)$. The origin of the $\mathrm{mHz}$ QPO is, however, unclear. Neither the Keplerian frequency model (van der Klis et al. 1987b) nor the beat frequency model (Alpar \& Shaham 1985) can explain the origin of these QPO in all systems. QPOs below the neutron star spin frequency cannot be explained by the Keplerian frequency model because if the Keplerian frequency at the magnetosphere is less than the spin frequency then the propeller mechanism would inhibit accretion (Stella et al. 1986). This is the case of $4 \mathrm{U}$ $0115+63$ and V0332+53. Likewise, the beat frequency model predicts a centrifugal inhibition threshold that it is at variance with the observations (Finger 1998). A third model, the magnetic disc precession model (Shirakawa \& Lai 2002), attributes the $\mathrm{mHz}$ QPO in X-ray pulsars to warping/precession modes induced by magnetic torques near the inner edge of the accretion disc. The applicability of this model to all sources is not as yet certain (Mukherjee et al. 2006).

Unlike LMXBs where the power spectral parameters and strength of the various noise components (anti)correlate with the mass accretion rate, $\dot{M}$, HMXB noise appears to depend weakly on $\dot{M}$ (we assume that the accretion rate increases/decreases during the rise/decay of the outburst), at least at high and intermediate accretion rates. Neither the characteristic frequencies nor the rms of the Lorentzians that account for the band-limited noise show a clear relationship with X-ray flux in the diagonal branch, despite the change of about 1.5 order of magnitude in luminosity (see Tables 3-5). The lack of such correlations were also noticed in the 1985 outburst of EXO 2030+375 (Angelini et al. 1989; Belloni \& Hasinger 1990).

$L_{\mathrm{S}}$ is exclusive of accreting X-ray pulsars and it is associated with the pulse noise. Its central frequency coincides with the frequency of the fundamental peak of the pulse period and suggests a strong coupling between the periodic and aperiodic noise components. This coupling implies that the instabilities in the accretion flow that give rise to the aperiodic variability must travel all the way down to the neutron star surface. The reader is referred to Lazzati \& Stella (1997) and Burderi et al. (1997) for a detailed study of the coupling between the periodic and aperiodic variability in X-ray pulsars and to Reig et al. (2006) for the particular case of $\mathrm{V} 0332+53 . L_{\mathrm{S}}$ is absent in the horizontal branch. In the case of $\mathrm{V} 0332+53$, the disappearance of the pulse peaks in the horizontal branch is accompanied by the disappearance of $L_{\mathrm{s}}$, which supports the idea of a common physical origin.

\subsection{Are there atoll and $Z$ sources in HMXBs?}

The correlated timing and spectral analysis defines two different types of Be/X-ray binaries. The differences are not only seen in the shape of the CD but also in the number and properties of the noise components. 

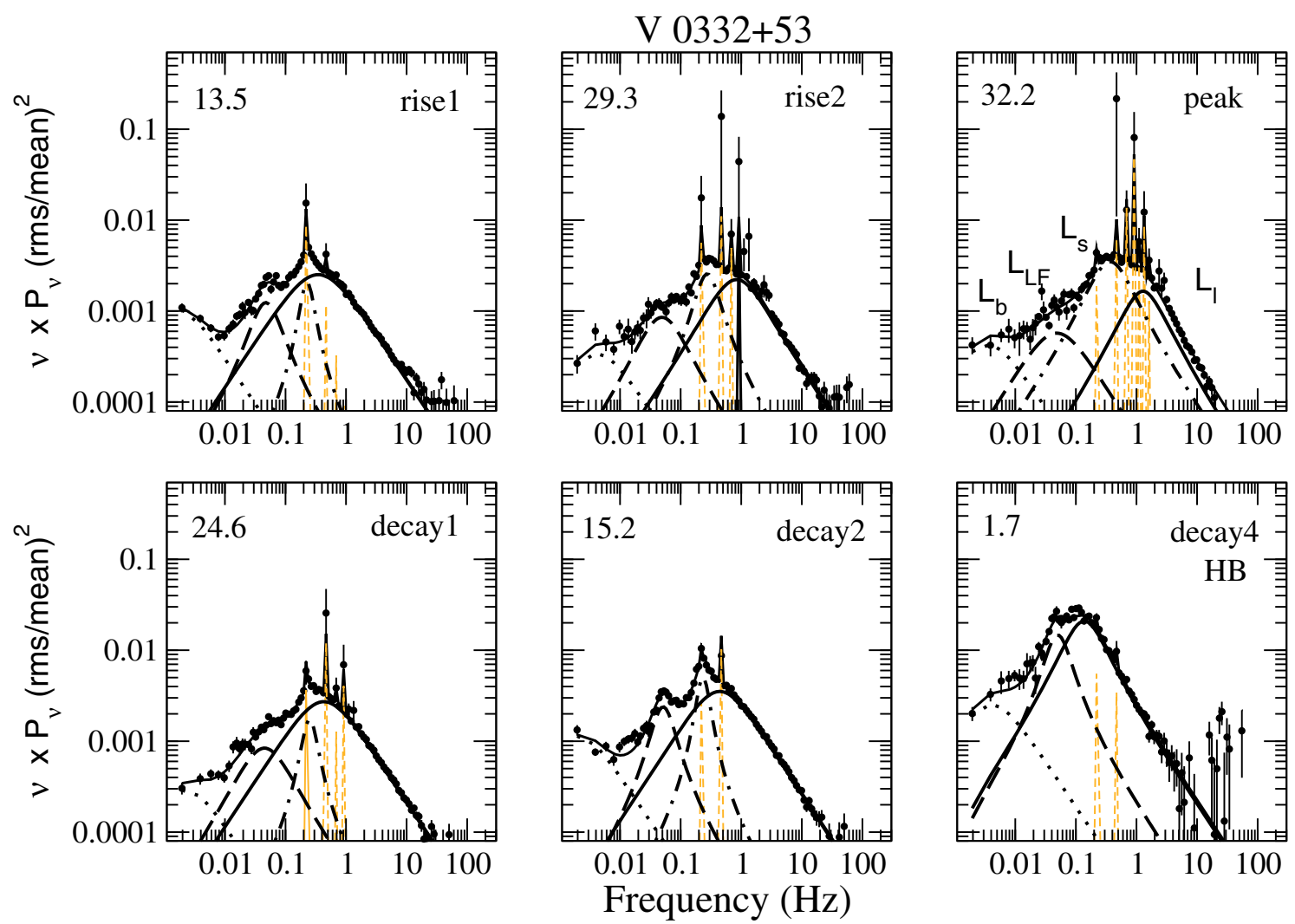

Fig. 9. Power spectra of V0332+53 and fit functions of various spectral regions. The number at the top left of each panel is the X-ray luminosity in units of $10^{37} \mathrm{erg} \mathrm{s}^{-1}$. HB stands for horizontal branch. The different lines indicate the individual Lorentzian components of the fit: $L_{\mathrm{b}}$ (dotted), $L_{\mathrm{l}}$ (thick solid black), $L_{\mathrm{LF}}$ (thick dashed), $L_{\mathrm{s}}$ (dashed-dotted), $L_{\mathrm{u}}$ (thin solid black). The pulse peaks (fundamental and harmonics) are represented by thin dashed orange lines.

The pattern traced by $\mathrm{V} 0332+53$ in the $\mathrm{CD}$ reminds that of the Z-sources, with the diagonal branch being the analogue to the normal branch and the HMXB horizontal branch branch being the counterpart of the LMXB horizontal branch. The flaring branch would be missing in $\mathrm{V} 0332+53$. As in $\mathrm{Z}$ sources, the hardest spectrum corresponds to a lower count rate state. Also, the fractional rms is higher and the characteristic frequencies are lower in the horizontal branch than in the diagonal branch.

In 4U 0115+63, KS 1947+300 and EXO 2030+375, the HB also corresponds to a low-intensity state and also displays the highest rms of the noise components. However, the HB in these three sources is softer than the diagonal branch. Likewise, the overall shape and motion along the branches differs from that seen in V0332+53 and LMXBs atoll sources. The HB in $4 \mathrm{U}$ 0115+63, KS 1947+300 and EXO 2030+375 corresponds to a low-intensity spectrally soft state not seen in other types of X-ray binaries.

Further similarities between $\mathrm{V} 0332+53$ and $\mathrm{Z}$ sources and 4 U $0115+63$, KS $1947+300$ and EXO 2030+375 and atolls sources are found in the shape of the very-low frequency noise $(\lesssim 0.1 \mathrm{~Hz})$. Figure 11 shows the power spectra of the HB and peak of the outburst for V0332+53 and KS $1947+300$. This figure should be compared to, e.g., Fig. 4 in van der Klis (1994). The noise below $\sim 0.1 \mathrm{~Hz}$ in the HB is flat-topped in KS $1947+300$ (and in EXO 2030+375 and 4U 0115+63), as in the island state of atoll sources, and power-law like in V0332+53, as in the $\mathrm{HB}$ of $\mathrm{Z}$ sources.

Initially, the differences between LMXB $Z$ and atoll sources were ascribed to a higher magnetic-field strength and higher accretion rates in $Z$ sources. However, the detection in atoll sources of QPOs that are reminiscent of HBO and NBO (Ford \& van der Klis 1998; Wijnands \& van der Klis 1999b) and the existence of sources that display the two types of behaviour, $Z$ and atoll (Homan et al. 2007a,b), question the validity of the proposed higher magnetic field in the $Z$ sources. Nevertheless, it is worth noting that $\mathrm{V} 0332+53$ displays the most intense outburst and contains the stronger magnetic field of the four sources. The peak luminosity in V0332+53 is about a factor 2,3 and 5 that of EXO 2030+375, 4U 0115+63 and KS 1947+300, respectively. The strength of the magnetic field can be estimated from the energy of cyclotron resonant scattering features (CRSF) through the relation

$E_{\mathrm{cyc}}=11.6 \frac{B}{10^{12} \mathrm{G}}(1+z)^{-1} \mathrm{keV}$

where $B$ is the magnetic field and $z$ is the gravitational redshift. As mentioned above, KS $1947+300$ is the only source for which no CRSF has been reported, while for $4 \mathrm{U} 0115+63$ $E_{\text {cyc }}=12 \mathrm{keV}$ (Santangelo et al. 1999), for EXO 2030+375 $E_{\mathrm{cyc}}=11 \mathrm{keV}$ (Wilson et al. 2008), and for V0332+53 $E_{\mathrm{cyc}}=$ $27 \mathrm{keV}$ (Pottschmidt et al. 2005). Thus the magnetic field in V0332+53 is about $2-3$ times stronger than in the other three systems.

It is tempting to attribute the differences between $\mathrm{Be} / \mathrm{X}$-ray binaries to the source brightness and magnetic field strength. The power-law noise at low frequencies in the HB of V0332+53 could be due to the higher intensity and the presence of $L_{\mathrm{S}}$ noise to a higher magnetic field. $4 \mathrm{U} 0115+63$, KS $1947+300$ and EXO $2030+375$ show flat-topped noise and lack the $L_{\mathrm{S}}$ component. However, with only one source of this type no definitive conclusion can be drawn about whether the source brightness and 
Table 5. Power spectral parameters (centroid frequency, $F W H M$ and rms) for the $L_{\mathrm{mQPO}}, L_{\mathrm{LF}}$ and $L_{\mathrm{s}}$ components. Errors are $90 \%$ confidence level. The centroid frequency of the zero-centred Lorentzian was fixed during the fit. HB stands for horizontal branch.

\begin{tabular}{|c|c|c|c|c|c|c|c|c|c|c|}
\hline $\begin{array}{l}\text { Spectral } \\
\text { region }\end{array}$ & $\begin{array}{c}L_{\mathrm{x}} \\
\times 10^{37} \\
\mathrm{erg} \mathrm{s}^{-1} \\
\end{array}$ & $\begin{array}{c}v_{\mathrm{mQPO}} \\
(\mathrm{Hz})\end{array}$ & $\begin{array}{c}L_{\mathrm{mQPO}} \\
F W H M_{\mathrm{mQPO}} \\
(\mathrm{Hz}) \\
\end{array}$ & $\begin{array}{c}\mathrm{rms}_{\mathrm{mQPO}} \\
(\%) \\
\end{array}$ & $\begin{array}{l}v_{\mathrm{LF}} \\
(\mathrm{Hz})\end{array}$ & $\begin{array}{c}L_{\mathrm{LF}} \\
F W H M_{\mathrm{LF}} \\
(\mathrm{Hz}) \\
\end{array}$ & $\begin{array}{c}\mathrm{rms}_{\mathrm{LF}} \\
(\%)\end{array}$ & $\begin{array}{c}v_{\mathrm{s}} \\
(\mathrm{Hz})\end{array}$ & $\begin{array}{c}L_{\mathrm{s}} \\
F W H M_{\mathrm{s}} \\
(\mathrm{Hz}) \\
\end{array}$ & $\begin{array}{r}\mathrm{rms}_{\mathrm{s}} \\
(\%)\end{array}$ \\
\hline \multicolumn{11}{|c|}{$4 \mathrm{U} 0115+63$} \\
\hline rise1 & 7.5 & - & - & - & 0 & $0.14 \pm 0.03$ & $10.7 \pm 0.7$ & - & - & - \\
\hline rise2 & 11.8 & - & - & - & $0.038 \pm 0.008$ & $0.06 \pm 0.01$ & $6.3 \pm 0.5$ & - & - & - \\
\hline peak & 11.0 & $3.7 \pm 0.4$ & $4.3 \pm 0.7$ & $8.8 \pm 0.4$ & $0.006 \pm 0.003$ & $0.09 \pm 0.01$ & $8.0 \pm 0.3$ & - & - & - \\
\hline decay 1 & 7.9 & $2.6 \pm 0.4$ & $5.5 \pm 1.0$ & $8.0 \pm 0.8$ & $0.049 \pm 0.006$ & $0.06 \pm 0.01$ & $7.3 \pm 0.6$ & - & - & - \\
\hline decay2 & 6.0 & - & - & - & $0.059 \pm 0.005$ & $0.06 \pm 0.01$ & $6.3 \pm 0.6$ & - & - & - \\
\hline decay3 & 5.0 & $3.6 \pm 0.6$ & $4.2 \pm 1.0$ & $5.4 \pm 0.3$ & $0.065 \pm 0.003$ & $0.05 \pm 0.01$ & $6.8 \pm 0.3$ & - & - & - \\
\hline decay4(HB) & 0.9 & - & - & - & 0 & $0.03 \pm 0.01$ & $12.8 \pm 1.0$ & - & - & - \\
\hline \multicolumn{11}{|c|}{ V0332+53 } \\
\hline rise1 & 13.5 & - & - & - & $0.038 \pm 0.003$ & $0.059 \pm 0.006$ & $4.7 \pm 0.1$ & $0.22 \pm 0.01$ & $0.12 \pm 0.01$ & $4.1 \pm 0.1$ \\
\hline rise2 & 29.3 & - & - & - & $0.029 \pm 0.003$ & $0.079 \pm 0.009$ & $4.4 \pm 0.1$ & $0.25 \pm 0.01$ & $0.26 \pm 0.03$ & $5.8 \pm 0.3$ \\
\hline peak & 32.2 & - & - & - & $0.010 \pm 0.009$ & $0.094 \pm 0.020$ & $4.1 \pm 0.6$ & $0.23 \pm 0.01$ & $0.57 \pm 0.03$ & $8.5 \pm 0.4$ \\
\hline decay 1 & 24.6 & - & - & - & $0.022 \pm 0.007$ & $0.078 \pm 0.009$ & $4.5 \pm 0.4$ & $0.24 \pm 0.01$ & $0.15 \pm 0.03$ & $4.0 \pm 0.3$ \\
\hline decay2 & 15.2 & - & - & - & $0.046 \pm 0.002$ & $0.043 \pm 0.003$ & $5.3 \pm 0.2$ & $0.23 \pm 0.01$ & $0.13 \pm 0.01$ & $6.2 \pm 0.1$ \\
\hline decay3 & 8.8 & - & - & - & $0.045 \pm 0.002$ & $0.053 \pm 0.005$ & $9.4 \pm 0.4$ & $0.23 \pm 0.01$ & $0.06 \pm 0.02$ & $3.2 \pm 0.9$ \\
\hline decay4(HB) & 1.7 & - & - & - & $0.043 \pm 0.005$ & $0.050 \pm 0.030$ & $14.4 \pm 0.6$ & - & - & - \\
\hline
\end{tabular}
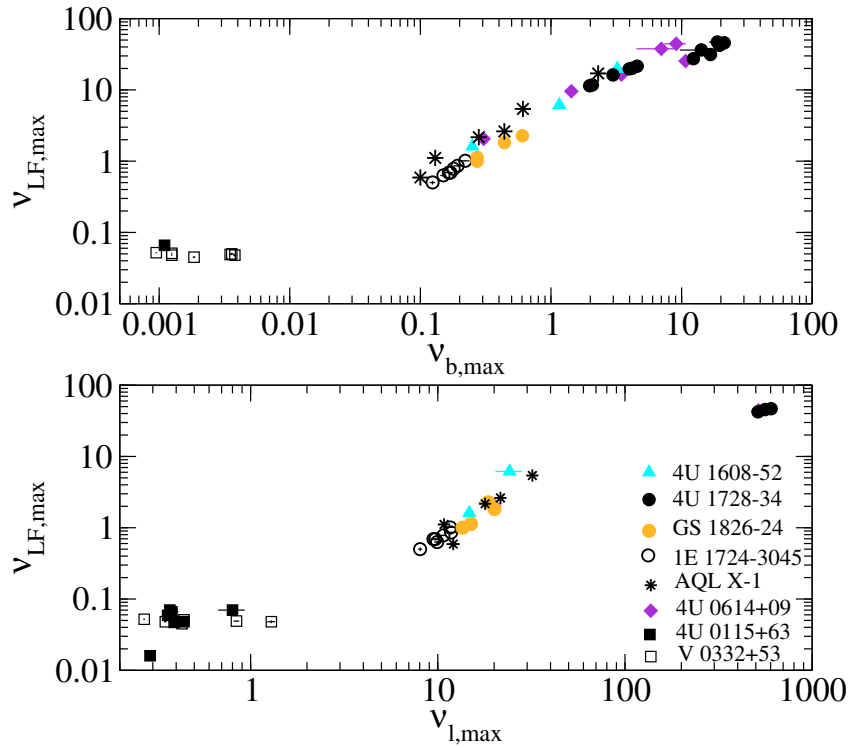

Fig. 10. Correlation between the characteristic frequencies of the noise components. $\mathrm{Be} / \mathrm{X}$ exhibit lower characteristic frequencies than LMXBs.

magnetic field strength allows the distinction between different types of systems among HMXB. Future observations will clarify this issue.

\section{Conclusions}

We have performed, for the first time, a systematic analysis of the spectral (through the study of hardness ratios) and rapid aperiodic variability of four $\mathrm{Be} / \mathrm{X}$-ray pulsars during type II outbursts. Our aim was to characterise the rapid aperiodic variability in correlation with the position in the colour-colour diagram as it has been done for black-hole systems and low-mass X-ray binaries.

The correlated spectral-timing behaviour in Be/X-ray binaries shares a number of similarities with low-mass X-ray binaries, namely, i) existence of spectral branches in the CD/HID. At high and intermediate flux the sources move along the diagonal
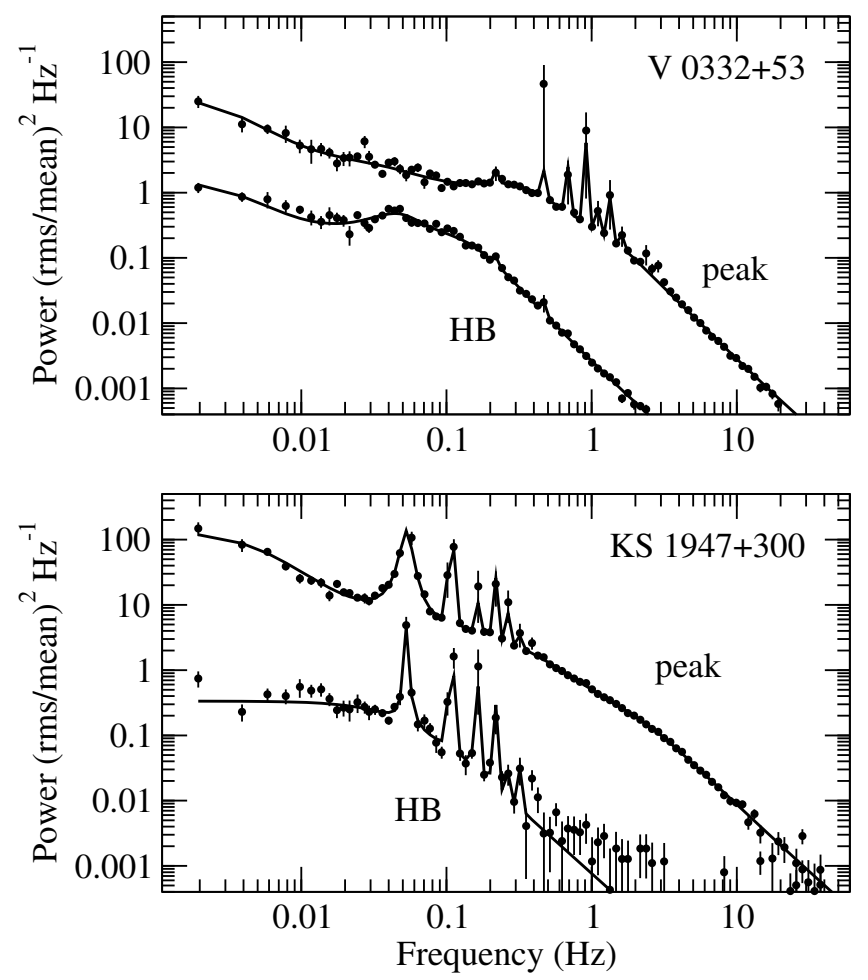

Fig. 11. Representative power spectra of high- and low-intensity states. Note the different shape of the noise at low frequencies. Flat-topped noise is seen in the HB of KS 1947+300 (and EXO 2030+375 and $4 \mathrm{U} 0115+63$ ) and power-law noise in $\mathrm{V} 0332+53$. Confront this figure with Fig. 4 of van der Klis (1994) and note the similarities between KS $1947+300$ and atoll sources and between V0332+53 and Z sources. The "peak" power spectrum was shifted by $\times 100$ for easy display.

branch; at very low count rate the soft colour decreases, while the hard colour remains fairly constant defining a horizontal branch; ii) smooth motion, i.e. without jumps, in the CD/HID; iii) the low-intensity states are more variable (in terms of fractional rms); iv) description of power spectra in terms of a small number of Lorentzian components; v) similar BLN namely, broad Lorentzians $Q \sim 0-0.3$ describing the low-frequency and highfrequency noise $\left(L_{\mathrm{b}}, L_{1}, L_{\mathrm{u}}\right)$ and peaked noise in between $\left(L_{\mathrm{LF}}\right)$ 
and vi) flat-topped noise at the lower frequencies in the horizontal branch that turns into power-law noise in the diagonal branch.

There are also important differences: i) different patterns in the $\mathrm{CD} / \mathrm{HID}$. Unlike low-mass X-ray binary and black hole systems, the low-intensity state is associated with a soft state (except for V0332+53), ii) slower motion along the spectral branches, hours to days in LMXBs, weeks to months in HMXBs, iii) the characteristic time scales implied by the noise components are about one order of magnitude longer in HMXBs (e.g. $\mathrm{mHz}$ QPOs), iv) $L_{\mathrm{S}}$ noise in HMXBs and v) no apparent correlation between the power spectral parameters (characteristic frequencies, rms) and mass accretion rate.

Although we have analysed all Be/X-ray binaries that showed type II outbursts in the period 1996-2007 and had good data coverage and sampling, the number of sources is not large enough to draw definite conclusions on the underlying physical parameters that may explain the differences between the two subgroups. Increasing the number statistics is, however, difficult given the transient nature of their X-ray emission and unpredictability of the type II outbursts.

Even though all four sources analysed in this work are X-ray pulsars, it is not clear whether the high-mass X-ray binaries with supergiant companions show a similar behaviour. In these systems accretion occurs via the strong stellar wind of the massive companion, which contrasts with accretion via a disc, as it is believed to occur in Be/X-ray binaries during outburst. The problem of this type of studies in supergiant X-ray binaries is that they are rather stable in long time scales and do not show a wide range in X-ray flux.

Acknowledgements. This research was supported by the European Union Marie Curie grant MTKD-CT-2006-039965. This research has made use of NASA's Astrophysics Data System Bibliographic Services and of the SIMBAD database, operated at the CDS, Strasbourg, France. The ASM light curve was obtained from the quick-look results provided by the ASM/RXTE team.

\section{References}

Angelini, L., Stella, L., \& Parmar, A. N. 1989, ApJ, 346, 906

Alpar, M. A., \& Shaham, J. 1985, Nature, 316, 239

Belloni, T., \& Hasinger, G. 1990, 230, 103

Belloni, T., Psaltis, D., \& van der Klis, M. 2002, ApJ, 572, 392

Belloni, T., Homan, J., Casella, P., et al. 2005, A\&A, 440, 207

Bernacca, P. L., Iijima, T., \& Stagni, R. 1984, A\&A, 132, L8

Borozdin, K., Gilfanov, M., Sunyaev, R., et al. 1990, Sov. Astron. Lett., 16, 345 Burderi, L., Robba, N. R., La Barbera, N., \& Guainazzi, M. 1997, ApJ, 481, 943 Camero Arranz, A., Wilson, C. A., Connell, P., et al. 2005, A\&A, 441, 261

Chakrabarty, D., Koh, T., Bildsten, L., et al. 1995, ApJ, 446, 826

Coburn, W., Kalemci, E., Kretschmar, P., et al. 2004, ATel, 337

Coe, M. J., Longmore, A. J., Payne, B. J., \& Hanson, C. G. 1987, MNRAS, 226, 455

Coe, M. J., Payne, B. J., Longmore, A., \& Hanson, C. G. 1988, MNRAS, 232, 865

Corbet, R. H. D., Charles, P. A., \& van der Klis, M. 1986, A\&A, 162, 117

Finger, M. H. 1998, AdSpR, 22, 1007

Finger, M. H., Wilson, R. B., \& Harmon, B. A. 1996, ApJ, 459, 288

Ford, E., \& van der Klis, M. 1998, ApJ, 506, L39

Galloway, D. K., Morgan, E. H., \& Levine, A. M. 2004, ApJ, 613, 1164

Goranskij, V., \& Barsukova, E. 2004, ATel, 245

Goranskii, V. P., Esipov, V. F., Lyutyi, V. M., \& Shugarov, S. Yu. 1991, Sov. Astron. Lett., 17, 399

Grankin, K. N., Shevchenko, V. S., \& Yakubov, S. D. 1991, Sov. Astron. Lett., 17,415

Hasinger, G. 1987, A\&A, 186, 153

Hasinger, G., \& van der Klis, M. 1989, A\&A, 225, 79

Hayasaki, K., \& Ozakaki, A. T. 2004, MNRAS, 350, 971

Heindl, W. A., Coburn, W., Gruber, D. E., et al. 1999, ApJ, 521, L49

Heindl, W. A., Rothschild, R. E., Coburn, W., et al. 2004, in X-ray Timing 2003: Rossi and Beyond. held 3-5 November, 2003 in Cambridge, MA. ed. P. Kaaret, F. K. Lamb, \& J. H. Swank (Melville, NY: American Institute of Physics), AIP Conf. Proc., 714, 323
Homan, J., van der Klis, M., Wijnands, R., Belloni, T., \& Fender, R. 2007a, ApJ, 656,420

Homan, J., Wijnands, R., Altamirano, D. \& Belloni, T. 2007b, ATel, 1165

Inam, S. Ç., Baykal, A., Swank, J., \& Stark, M. J. 2004, ApJ, 616, 463

Jernigan, J. G., Klein, R. I., \& Arons, J. 2000, ApJ, 530, 975

Kaur, R., Paul, B., Raichur, H., \& Sagar, R. 2007, ApJ, 660, 1409

Klochkov, D., Horns, D., Santangelo, A., et al. 2007, A\&A, 464, L45

Kriss, G. A., Cioffi, D. F., \& Canizares, C. R. 1983, ApJ, 272, 439

Lazzati, D., \& Stella, L. 1997, ApJ, 476, 267

Leahy, D. A., Darbro, W., Elsner, R. F., et al. 1983, ApJ, 266, 160

Levine, A., \& Corbet, R. 2000, IAUC, 7524

Makishima, K., Mihara, T., Ishida, M., et al. 1990, ApJ, 365, L59

Markert, T. H., Canizares, C. R., Clark, G. W., et al. 1977, ApJ, 218, 801

Mavromatakis, F. 1994, A\&A, 285, 209

Miyamoto, S., Kimura, K., Kitamoto, S., Dotani, T., \& Ebisawa, K. 1991, ApJ, 383,784

Mowlavi, N., Kreykenbohm, I., Shaw, S. E., et al. 2006, A\&A, 451, 187

Motch, C., \& Janot-Pacheco, E. 1987, A\&A, 182, L55

Motch, C., Stella, L., Janot-Pacheco, E., \& Mouchet, M. 1991, ApJ, 369, 490

Mukherjee, U., Bapna, S., Raichur, H., Paul, B., \& Jaaffrey, S. N. A. 2006, JApA, 27,25

Nakajima, M., Mihara, T., Makishima, K., \& Niko, H. 2006, ApJ, 646, 1125

Nowak, M. A. 2000, MNRAS, 318, 361

Nowak, M. A., Vaughan, B. A., Wilms, J., Dove, J. B., \& Begelman, C. 1999, ApJ, 510, 874

Naik, S., Callanan, P. J., Paul, B., \& Dotani, T. 2006, ApJ, 647, 1293

Negueruela, I., Roche, P., Fabregat, J., \& Coe, M. J. 1999, MNRAS, 307, 695

Negueruela, I., Israel, G. L., Marco, A., Norton, A. J., \& Speziali, R., 2003, A\&A, 397, 739

Okazaki, A., \& Negueruela, I. 2001, A\&A, 377, 161

Olive, J., Barret, D., \& Gierlinski, M. 2003, ApJ, 583, 416

Parmar, A. N., White, N. E., Stella, L., Izzo, C., \& Ferri, P. 1989, ApJ, 338, 359

Pottschmidt, K., Wilms, J., Nowak, M., et al. 2003, A\&A, 407, 1039

Pottschmidt, K., Kreykenbohm, I., Wilms, J., et al. 2005, ApJ, 634, L97

Priedhorsky, W., Hasinger, G., \& Lewin, W. H. G 1986, ApJ, 306, L91

Psaltis, D., Belloni, T., \& van der Klis, M. 1999, 520, 262

Qu, J. L., Zhang, S., Song, L. M., \& Falanga, M. 2005, ApJ, 629, L33

Rappaport, S., Markert, T., Li, F. K., et al. 1977, ApJ, 217, L29

Rappaport, S., Clark, G. W., Cominsky, L., Li, F., \& Joss, P. C. 1978, ApJ, 224, L1

Reig, P. 2007, MNRAS, 377, 867

Reig, P., \& Coe, M. J. 1998, MNRAS, 294, 118

Reig, P., \& Coe, M. J. 1999, MNRAS, 302, 700

Reig, P., Fabregat, J., \& Coe, M. J. 1997, A\&A, 322, 193

Reig, P., Stevens, J. B., Coe, M. J., \& Fabregat, J. 1998, MNRAS, 301, 42

Reig, P., van Straaten, S., \& van der Klis, M. 2004, ApJ, 602, 918

Reig, P., Martínez-Núñez, S., \& Reglero, V. 2006, A\&A, 449, 703

Reig, P., Larionov, V., Negueruela, I., Arkharov, A. A., \& Kudryavtseva, N. A. 2007, A\&A, 462, 1081

Reynolds, A. P., Parmar, A. N., \& White, N. E. 1993, ApJ, 414, 302

Santangelo, A., Segreto, A., \& Giarrusso, S. 1999, ApJ, 523, L85

Schulz, N. S., Hasinger, G., \& Truemper, J. 1989, A\&A, 225, 48

Shirakawa, A., \& Lai, D. 2002, ApJ, 565, 1134

Stella, L., White, N. E., Davelaar, J., et al. 1985, ApJ, 288, L45

Stella, L., White, N. E., \& Rosner, R. 1986, ApJ, 308, 669

Stollberg, M. T., Finger, M. H., Wilson, R. B., et al. 1999, ApJ, 512, 313

Sun, X. J., Li, T. P., Wu, M., \& Cheng, L. X. 1994, A\&A, 289, 127

Swank, J., \& Morgan, E. 2000, IAUC, 7532

Takeshima, T., Dotani, T., Mitsuda, K., \& Nagase, F. 1994, ApJ, 436, 871

Tanaka, Y. 1983, IAUC, 3891

Terrell J., \& Priedhorsky, W. C. 1984, ApJ, 285, L15

van der Klis, M. 1989, in Timing Neutron stars, ed. H. Ögelman, \& E. P. J. van den Heuvel, NATO ASI ser., C, 262, 27

van der Klis, M. 1994, ApJSS, 92, 511

van der Klis, M. 2006, in Compact Stellar X-ray sources, ed. W. H. G. Lewin, \& M. van der Klis (Cambridge University Press), 39

van der Klis, M., Jansen, F., van Paradijs, J., et al., 1987a, ApJ, 313, L19

van der Klis, M., Stella, L., White, N., Jansen, F., \& Parmar, A. N. 1987b, ApJ, 316,411

van Straaten, S., van der Klis, M., di Salvo, T., \& Belloni, T. 2002, ApJ, 568, 912 van Straaten, S., van der Klis, M., \& Méndez, M. 2003, ApJ, 596, 1155

Wilson, C. A., Finger, M. H., Coe, M. J., Laycock, S., \& Fabregat, J. 2002, ApJ, 570,287

Wilson, C. A., Finger, M. H., \& Camero Arranz, A. 2008, ApJ, 678, 1263

White, N. E., \& Marshall, F. E. 1984, ApJ, 281, 354

Whitlock L. 1989, ApJ, 344, 371

Wijnands, R., \& van der Klis, M. 1999a, ApJ, 514, 939

Wijnands, R., \& van der Klis, M. 1999b, ApJ, 522, 965 